\title{
Characterization of On-Orbit GPS Transmit Antenna Patterns for Space Users
}

\author{
Jennifer E. Donaldson, Joel J. K. Parker, Michael C. Moreau, Goddard Space Flight Center, NASA \\ Dolan E. Highsmith, Philip Martzen, The Aerospace Corporation
}

\begin{abstract}
The GPS Antenna Characterization Experiment (GPS ACE) has made extensive observations of GPS L1 signals received at geosynchronous (GEO) altitude, with the objective of developing comprehensive models of the signal levels and signal performance in the GPS transmit antenna side lobes. The experiment was originally motivated by the fact that data on the characteristics and performance of the GPS signals available in GEO and other high Earth orbits was limited. The lack of knowledge of the power and accuracy of the side lobe signals on-orbit added risk to missions seeking to employ the side lobes to meet navigation requirements or improve performance. The GPS ACE Project filled that knowledge gap through a collaboration between The Aerospace Corporation and NASA Goddard Spaceflight Center to collect and analyze observations from GPS side lobe transmissions to a satellite at GEO using a highly-sensitive GPS receiver installed at the ground station. The GPS ACE architecture has been in place collecting observations of the GPS constellation with extreme sensitivity for several years. This sensitivity combined with around-the-clock, all-in-view processing enabled full azimuthal coverage of the GPS transmit gain patterns over time to angles beyond 90 degrees off-boresight. Results discussed in this paper include the reconstructed transmit gain patterns, with comparisons to available pre-flight gain measurements from the GPS vehicle contractors. For GPS blocks with extensive ground measurements, the GPS ACE results show remarkable agreement with ground based measurements. For blocks without extensive ground measurements, the GPS ACE results provide the only existing assessments of the full transmit gain patterns. The paper also includes results of pseudorange deviation analysis to assess systematic errors associated with GPS side lobe signals.
\end{abstract}

\section{Introduction}

This manuscript describes an exhaustive dataset collected over a period of several years by the GPS Antenna Characterization Experiment (ACE) and the meticulous post-processing campaign to construct detailed maps of gain and pseudorange deviations for each of the satellites in the current GPS constellation. The antenna pattern maps resulting from this work provide verification of ground measurements of GPS satellite antenna gain patterns, and are expected to greatly facilitate the analysis of GPS performance for future Geosynchronous Orbit (GEO) or other High Earth Orbit (HEO) space applications.

There are a number of examples in recent years of operational space missions utilizing GPS measurements in GEO and $\mathrm{HEO}$, and the demonstrated navigation performance for these missions has greatly exceeded pre-launch expectations in many cases $[1,2,3]$. These missions realize such exceptional performance for several reasons: transmitted GPS power levels exceed formal specifications of performance; receiver technology has evolved to reliably track very weak GPS signals; and transmissions from the GPS satellite antenna side lobes, which are completely excluded from performance specifications, contribute significantly to signal availability and improve the geometry of tracked satellites. As a result, most receiver performance simulation and testing for these applications has been extremely conservative.

Space navigators have long sought to better understand the actual on-orbit performance of the GPS system available to HEO users, but publicly available data, particularly related to the performance of side lobe signals, has been 
extremely limited. In 2006, NASA, U. S. Air Force (USAF), and Aerospace Corporation personnel worked together to document Space Service Volume requirements for GPS III, establishing a minimum specification for signal strength and availability of main-lobe GPS transmissions [4]. This formal expansion of the GPS performance regime to a space service volume (SSV) was a positive development - this addressed the risk that many space missions already utilizing GPS in these orbital regimes were doing so without formal requirements governing future performance. Unfortunately, this specification defined conservative performance requirements based on the worst performing satellites in the GPS constellation at the time, without considering contributions from side lobe transmissions. As a result, the specified signal availability documented in the formal GPS III requirements vastly under-estimates the number of signals demonstrated to be available on recent missions.

In 2015, Lockheed Martin publicly released measurements of Block IIR and IIR-M transmit antenna patterns collected during pre-launch testing [5]. This provided a detailed look at signal variations as a function of azimuth and elevation for all of the Lockheed-built IIR and IIR-M satellites. In spite of this excellent dataset, there is still no comparable publicly available dataset on the Block II/IIA/IIF satellites, or future GPS satellites. Moreover, questions still remained regarding how well the ground measurements matched actual on-orbit transmissions, and what measurement errors a user might experience when using side lobe signals.

The GPS Antenna Characterization Experiment began as a research collaboration between The Aerospace Corporation and NASA Goddard Space Flight Center (GSFC) to conduct a comprehensive characterization of the on-orbit GPS L1 transmit antennas. In this unique experimental set-up, GPS signals are received by a satellite in a GEO orbit and relayed to the GPS receiver and processing equipment located in the satellite ground station. In this manner, the GEO satellite serves as a bent-pipe relay, delivering the GPS signals to the user on the ground, not unlike the way many RF communications relay satellites work. Two GPS receivers are implemented in the ground station: a software version of the Goddard Navigator GPS receiver, with a sensitivity of approximately $22-25 \mathrm{~dB}-\mathrm{Hz}$ [6]; and the Aerospace Mariposa GPS Receiver (MGPSR), which through the use of deeply-aiding tracking techniques is able to record GPS signals as low as $0 \mathrm{~dB}-\mathrm{Hz}[7]$. The combination of the extremely weak sensitivity of the MGPSR and the operation of the experiment for multiple years has resulted in an exhaustive collection of GPS measurements spanning nearly every azimuth and elevation angle combination for each GPS satellite, enabling an in-depth analysis of the on-orbit performance of the GPS transmissions available to space users. This manuscript provides a high-level overview of the experiment configuration and describes the comprehensive post-processing campaign, including link budget error models and techniques employed to identify and remove outliers in the dataset. Finally, the resulting antenna gain and pseudorange deviations results are presented.

\section{Previous Work to Characterize GPS Signals in Space}

Some of the earliest references regarding the use of GPS for GEO satellites are publications by Jorgensen in 1982 [8] and $\mathrm{Wu}$ et al. in 1992 [9]. Figure 1 illustrates the geometry associated with GPS signal transmissions available to users in HEO. Strong GPS signals emanating from greater than 13.8 degrees off-boresight pass by the limb of the Earth and may be received by satellites on the opposite side of the Earth, but many weaker signals from the side lobes may also be available. Also noted in this figure is the 23.5 degree limit of the SSV specification for L1 signals, which corresponds approximately with the null between the main lobe and the first side lobe of most GPS satellites. In 2001, Moreau's thesis [10] presented a GPS receiver architecture for navigation in this regime, and around this time results from a number of early flight experiments and applications became available. This included Falcon Gold 1997 [11], Equator-S in 1998 [12], AMSAT OSCAR-40 in 2000 [13], and a paper by Kronman describing a bent-pipe GPS navigation system used in conjunction with a GEO satellite [14]. These early experiments, while generally successful, provided only a very limited amount of data on actual GPS signal levels. Nevertheless, there were sufficient data, particularly from the NASA-sponsored AMSAT OSCAR-40 experiment, to make it clear that significant variations existed in transmitted power levels between different blocks of GPS satellites. The AMSAT experiment also provided indications that some side lobes signals were significantly stronger than predicted from the limited data available at the time. These insights prompted NASA personnel who were evaluating the application of GPS to HEO missions to re-evaluate the applicable GPS performance requirements.

In this time frame, the official GPS specifications relating to signal strength and availability extended only to the Earth's limb. The main transmitting beam of the GPS satellite L1 antennas was typically assumed to measure a 


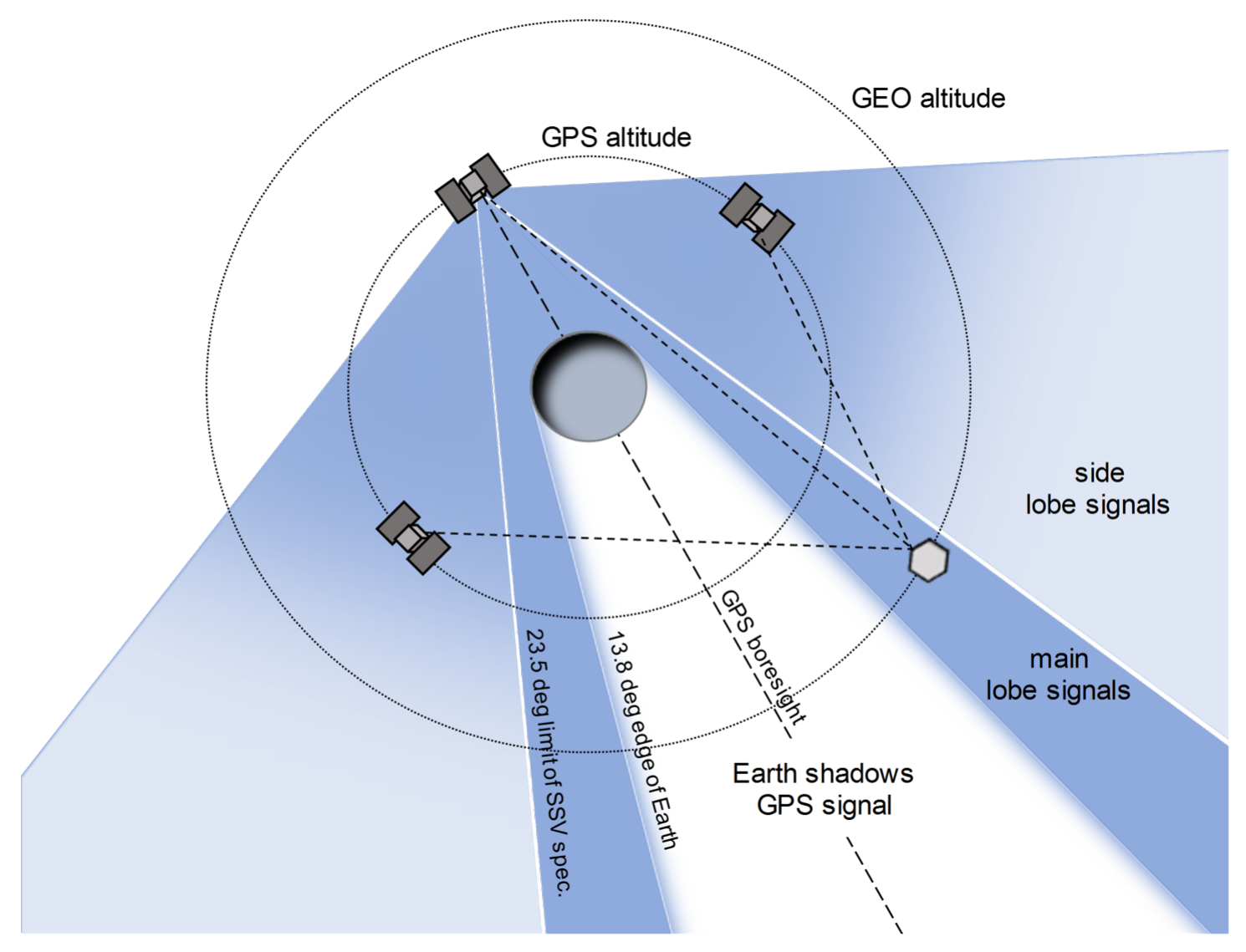

Figure 1: The geometry for reception of GPS signals in high Earth orbits.

half-power angle of 21.3 degrees [15], but there was very little published information regarding the GPS transmissions outside of a 14.3 degree half-angle, or possible variations in the shape of the GPS transmitter antennas, particularly in the side lobes. For many years, the only published data on the GPS L-band antenna pattern consisted of a paper by Czopek published in 1993 [16] that included four azimuth cuts of the relative gain from a Block II antenna. Data from the AMSAT experiment provided evidence that the main lobes of the Block IIR satellites were actually slightly narrower than the Block II/IIA patterns, and that at least some side lobe transmissions from Block IIR satellites were significantly stronger than what had been expected based on the Czopek data [13]. These observations were subsequently confirmed through data provided by Lockheed Martin, collected as part of the ground test program for the Block IIR and IIR-M antenna panels prior to launch. However, these data were not made available to the general user community until 2015 [5].

The insights gained through the AMSAT experiment demonstrated a need to better understand GPS signals available to space users and to consider documenting formal requirements for this performance. In response, Bauer et al. proposed metrics for GPS signal performance requirements in two SSV regimes: medium altitudes from 3,000 $\mathrm{km}$ to $8,000 \mathrm{~km}$, and high/geosynchronous altitudes from 8,000 km to 36,000 km [4]. More recently, GPS has been utilized by a wide number of operational HEO GPS applications [1, 2, 17, 18]. Each of these missions have demonstrated that through exploitation of transmissions from the side lobes of the GPS antenna pattern, the number of usable signals for navigation far exceeds what is predicted by the formal SSV requirements. NASA has continued to work with USAF to re-evaluate the GPS SSV requirements and consider whether revisions should be made to better reflect the performance currently realized by a number of civilian and military space applications of GPS [19]. However, in the time frame leading up to the acquisition of the GPS IIIF program, a consensus to adopt changes to the existing requirements could not be reached. 
Prior to the analysis of the GPS ACE data set, the best source of information on the GPS satellite gain patterns is the dataset on the Block IIR and IIR-M satellites described by Marquis [5]. A much more limited set of ground based antenna pattern measurements are available for the Block IIF satellites, but the side lobe measurements are only available for four azimuthal cuts through the antenna pattern, and the data are not publicly available. The European Space Agency published results from the GIOVE-A GPS experiment in 2013 [20], and a more exhaustive analysis of these results in 2017 [21]. The receiver on the GIOVE-A satellite performed up to 10 millisecond integrations and was able to record a large number of measurements of the stronger side lobe signals, providing limited insight into Block IIA and IIF antenna patterns for the first time.

\section{GPS ACE Project Description}

The GPS ACE collaboration between Aerospace and NASA GSFC began through respective independent research and development efforts, authorized though a NASA Space Act Agreement. The project began with significant receiver development efforts on both sides. Aerospace produced MGPSR, an ultra-weak signal tracking software defined radio receiver described in Section 3.2. NASA contributed two versions of its Navigator GPS Space Receiver platform [6]: a software defined radio version of the Navigator receiver flight software, and a version of the same receiver algorithms implemented in a commercial off the shelf (COTS) FPGA development board. The Aerospace MGPSR and Navigator receivers are complementary - the MGPSR is a highly-aided, near-real-time receiver suitable for implementation as part of a navigation ground architecture, while the Navigator is an on-board, unaided receiver designed for autonomous, real-time navigation applications.

Once the receivers were built and tested, they were installed at a GEO satellite ground station and configured to track the GPS L1 signals received by the GEO satellite and relayed to the ground station in a bent-pipe architecture. The goal of the project was to track the GPS L1 transmitters over a period of time sufficient to produce a data set of GPS $C / N_{0}$ and pseudorange measurements spanning every GPS transmitter azimuth and elevation angle combination for each GPS satellite in orbit. Ultimately, this data set would be used to recreate the GPS transmit antenna gain patterns and generate pseudorange deviation assessments for all of the GPS satellites from Blocks IIA, IIR, IIR-M, and IIF.

\subsection{GPS ACE Project Architecture}

The GPS ACE system architecture is depicted in Figure 2. Beginning at the top left, the GPS vehicles transmit L1 $\mathrm{C} /$ A signals at $1575.42 \mathrm{MHz}$. These signals are received at the GEO spacecraft with a GPS patch antenna. A sample bandwidth centered around L1 is transponded to the ground station on a telemetry channel dedicated to GPS, such that the GPS L1 spectrum is continuously available at the station. The signal is downconverted to an intermediate frequency (IF) and digitized into packets. The autonomous GPS receivers pull the packetized data from a local network, process the data and output GPS observation files containing pseudorange, carrier and carrier-to-noise ratio measurements. Multiples of each receiver type are installed to permit flexibility and redundancy.

Given the continuous availability of the GPS L1 spectrum at the ground station, the GPS ACE receivers produce daily observation files with a full 24 hours of tracking down to the sensitivity of the respective receivers: $22 \mathrm{~dB}-\mathrm{Hz}$ for the NASA Navigator at 20 msec integrations and less than $0 \mathrm{~dB}-\mathrm{Hz}$ for the Aerospace MGPSR at 30 sec integrations. The extreme sensitivity of the MGPSR enables reconstruction of the GPS transmit gain patterns deep into the side lobes, to beyond 90 deg off-boresight. The following subsections describe the MGPSR and Navigator GPS receivers in more detail. 


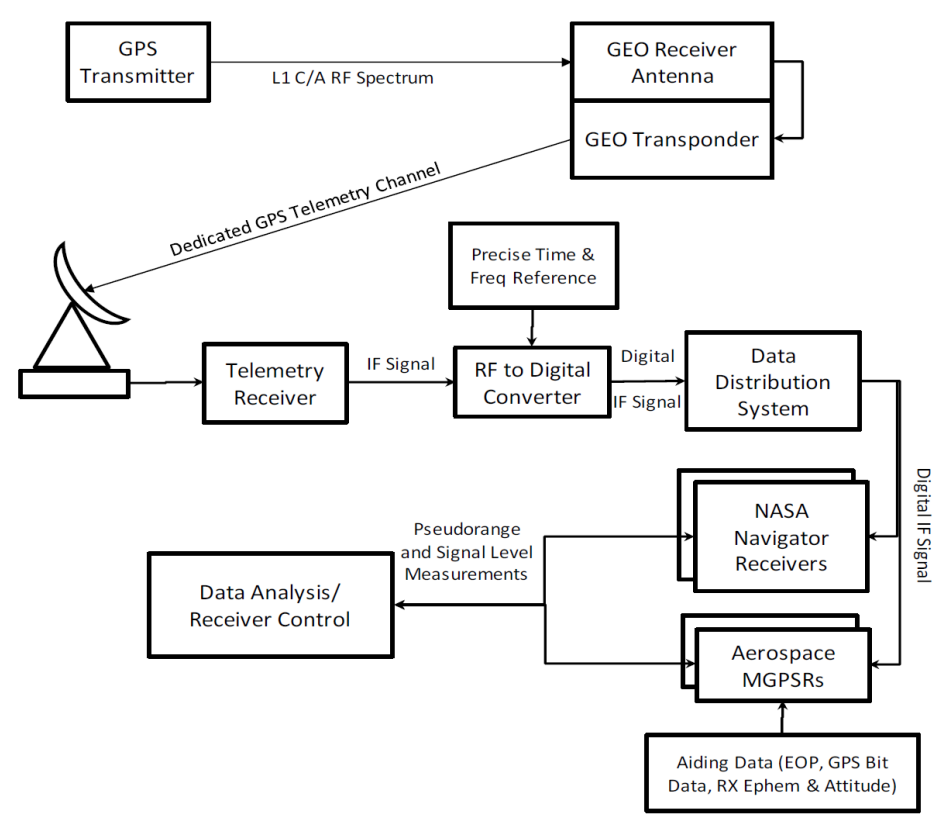

Figure 2: GPS ACE data collection architecture.

\subsection{Mariposa GPS Receiver (MGPSR) Algorithm}

The Mariposa GPS Receiver (MGPSR) is an Aerospace Corporation-developed ground-based software receiver that uses a highly aided, long integration batch processing algorithm. It was developed to operate in a non-real-time ground environment and process low signal-to-noise ratio (SNR) digital samples of GPS RF collected at orbital altitudes. Its roots derive from the data processing of the U. S. Air Force Academy-sponsored Falcon Gold experiment that was flown in November 1997 to measure GPS signals at high orbital altitudes [11]. The Falcon Gold data set, collected in a geosynchronous transfer orbit, contributed to the MGPSR design objectives and was used in work at the University of Colorado [22].

The current algorithm and design approach derive from orbit determination applications involving weak signal bentpipe GPS in an aided post-processing mode. In this mode, the GPS base-band data is captured and queued for processing a short time later when the following aiding information is available: a) GPS ephemeris and clock, b) approximate transponder ephemeris, and c) raw GPS subframe bit data. The subframe bit data effectively enables data wiping and long coherent processing, while good ephemeris and clock enable reduced search. The aiding information is combined to form the parameters of the expected received GPS signal. A paper providing a detailed description of this receiver and preliminary results from the GPS ACE experiment was presented as part of the US Government-only session of the Joint Navigation Conference in 2015 [7].

\subsubsection{Long Coherent Integration Method}

The objective of the MGSPR is to determine the model parameter errors based on signal samples. This allows the determination of the raw pseuodrange. The model error is found by searching for adjustments which maximize the correlation between the received signal and a signal replica. The MGPSR generates a sequence of replica samples corresponding to the digitized samples of the received signal.

In this application, the received signal and the replica have a differential delay and differential frequency offset. The objective of the correlation process is to estimate the SNR of the received signal and the differential time delay, $\tau$, between the signal and the replica. Long correlations are required to obtain accurate estimates of these parameters 
at low SNR values. The problem is compounded by the frequency difference between the received signal and the replica. As explained in [23], it is required to jointly estimate the time delay and the frequency difference in order to adequately obtain either. This is accomplished by computing the complex ambiguity function (CAF) shown in Eq. 1.

$$
C A F(\tau, \delta f)=\sum_{k=0}^{n} y_{k} r_{k+i}^{*} e^{-j 2 \pi k \delta f \Delta t}
$$

In this expression, $y_{k}$ and $r_{k+i}^{*}$ represent the sequence of signal and replica samples, respectively; $\tau$ is the differential time delay; $\delta f$ is the frequency offset; $\Delta t$ is the sample interval; and $i=\tau / \Delta t$ is the shift between the signal and replica sample index. The $\tau$ and $\delta f$ parameters are searched simultaneously for the $|C A F(\tau, \delta f)|$ peak. If a signal is present, a CAF correlation peak will be found at some time $\tau$ and Doppler $\delta f$ offset. Then to first order the measured raw pseudorange is $P R_{\text {raw }}=t_{5}-\left(t_{s v}+\tau\right)$. Here $t_{5}$ is the time of a representative sample within the correlation interval, and $t_{s v}$ is the corresponding modeled a priori SV pseudo-random noise (PRN) code phase time.

The MGPSR correlator performs long coherent integration by coherently combining Fast Fourier Transform (FFT) generated 1-msec complex correlations using methods similar to those described in references [24, 25]. For example, for 30-sec coherent processing, a set of 30,000 1-msec complex correlations are computed and stored. This 2-D array of 1-msec complex correlations is called a pre-CAF (PCAF). Some sub-set of the 1-msec correlation output is stored in each row of the PCAF depending on how well the time offset is known. The PCAF is computed with $\delta f=0$.

The PCAF rows are coherently combined, rotating by $\delta f$ to synthesize a frequency offset, forming a long integration $C A F(\tau, \delta f)$ result in the $(\tau, \delta f)$ search space. The objective is to find the $\tau_{p}, \delta f_{p}$ corresponding to the largest $|C A F(\tau, \delta f)|$. Once the CAF grid peak is located, an interpolation is performed to refine the peak location and obtain the output parameters. The method is effective when the frequency offset is much less than $1 \mathrm{kHz}$. At frequency offsets of $250 \mathrm{~Hz}$ or more, there is increasing power loss.

\subsection{2 $C / N_{0}$ Estimation}

The received carrier-to-noise ratio $C / N_{0}$ of the received GPS signal is computed in the MGPSR as

$$
C / N_{0}=10 \log _{10}((P-1) / T)
$$

where $P$ is the signal-to-noise power ratio given by

$$
P=\frac{|C A F(\tau, \delta f)|^{2}}{n \sigma^{2}}
$$

$T$ is the integration time in seconds, $\sigma^{2}$ is the sample variance, and $n$ is the number of samples. When no signal is present, the expected value for $P$ is $P=1$. These $C / N_{0}$ values are the measurements used in reconstructing the GPS transmit antenna patterns. The sample variance is computed as

$$
\sigma^{2}=\frac{1}{n} \Sigma\left(I^{2}+Q^{2}\right)
$$

which is essentially a measurement of the wide-band power. Since the measurements are generated based on a 30 second integration, there are $187.5 \times 10^{6}$ samples per correlation.

\subsection{Navigator GPS Receiver}

The NASA GSFC-developed Navigator GPS receiver is an L1 C/A, fast-acquisition, weak-signal-tracking spaceborne GPS receiver for high-altitude applications. It employs a fast Fourier transform (FFT)-based acquisition algorithm 
adapted from [24] and described in detail in [6]. This method allows the receiver to acquire weak GPS signals as low as $25 \mathrm{~dB}-\mathrm{Hz}$ in real-time with no a priori information to aid the receiver. Additionally, the receiver uses a 20-ms long coherent integration to track weak signals as low as $22 \mathrm{~dB}-\mathrm{Hz}$.

At the time that GSFC began development of the Navigator in the early 2000s, there existed few commercially available GPS receivers capable of operating reliably above low Earth orbit. GSFC had previously developed a GPS receiver called PiVoT based on a commercially available GPS chipset development kit. PiVoT incorporated customized software modifications that supported its use in HEO mission scenarios, and the receiver was used extensively in hardware in-the-loop testing and technology development activities. The PiVoT hardware did not support a path to flight qualification, which led to the development of Navigator as a new space-flight qualified HEO receiver. The first flight of the Navigator receiver was in 2009 as a technology demonstration on the Space Shuttle as part of the Hubble Space Telescope Servicing Mission 4 (HSM-4). It was first used operationally on NASA's Global Precipitation Measurement (GPM) mission in LEO, and subsequently Navigator was used for the on-board navigation system on the NASA Magnetospheric Multiscale (MMS) mission. The MMS mission features four spacecraft in highly elliptical orbits, and the Navigator receiver has demonstrated robust GPS performance at ranges from the Earth reaching nearly half way to the Moon [3]. The Navigator algorithms have been incorporated into the GPS receiver on the NASA Orion Crew Vehicle, and the design has been licensed to several commercial vendors of GPS receivers.

For the GPS ACE project, GSFC delivered two versions of the Navigator receiver, a software defined radio version of Navigator that runs in real-time on a Linux desktop computer and a re-programmable FPGA version of the receiver that runs on a COTS FPGA development board. The Navigator receiver was primarily used for real-time orbit determination experiments, which are not discussed in this paper, and for verification of the MGPSR measurements. The MGPSR was used for the antenna pattern reconstruction because of the lower $C / N_{0}$ sensitivity.

\section{Antenna Pattern Reconstruction}

The GPS ACE measurements from the MGPSR are processed to reconstruct transmit antenna patterns for all observed GPS spacecraft. The objective is to precisely map individual received power measurements and other observed signal characteristics back to the corresponding azimuth and elevation angle of the GPS transmitter, such that GPS signal characteristics may be documented on a per-satellite basis. Due to the geometry between the 24 hour GEO orbits and the 12 hour GPS orbits, as well as the yaw of the GPS spacecraft, the transmit patterns slowly fill in over time. If there were no gaps in tracking, the patterns would be completely filled in with measurements after approximately six months. Figure 3 shows how the patterns fill in over time.

Approximately two years of MGPSR measurements (non-continuous) were aggregated for post-processing and reconstruction of antenna gain and pseudorange deviations. Care was taken to exclude periods of time during which variations in the transmitted power levels from the GPS satellites were possible, such as during the L1 C/A power testing activities announced on January 25, 2017 [26]. A sample of just one day of MGPSR 30-sec observations from a GEO spacecraft is shown in Figure 4, which illustrates the extent of received signal levels and GPS off-boresight angles available in a single day of data.

The primary external tool used in the MGPSR data processing is the Orbit Determination Toolbox (ODTBX), an open source, MATLAB-based, orbit determination analysis tool developed at NASA GSFC [27]. ODTBX provides a variety of measurement simulation, estimation, and data visualization capabilities useful for early-phase mission analysis, including GPS pseudorange measurement models and a suite of GPS data analysis functions. The GPS models provide the ability to model physical parameters of the transmitters and receiver (relative geometry and antenna patterns), perform link budget analyses to estimate either transmit or received power, and a full suite of plotting and visualization routines. These latter capabilities were the basis of much of the geometric and link budget analysis performed on the GPS ACE data. 


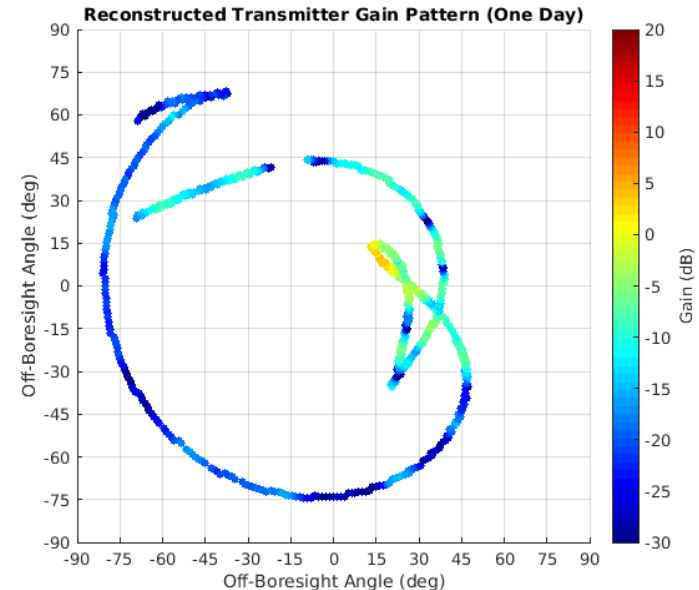

(a)

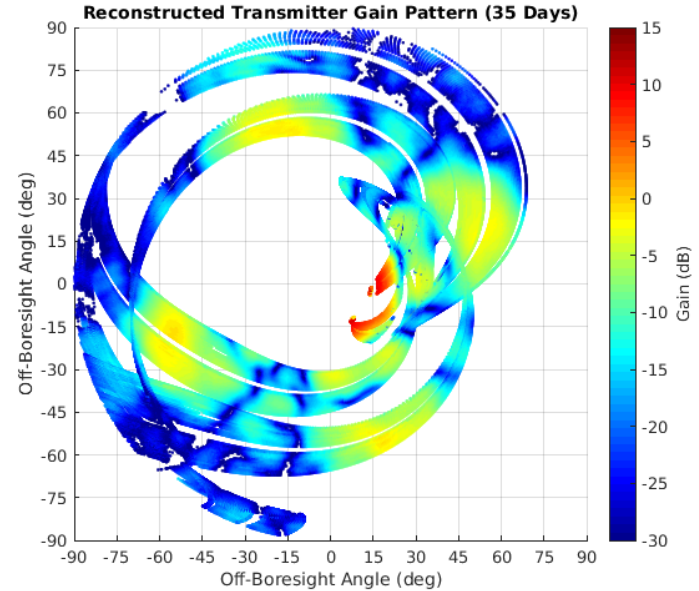

(b)

Figure 3: Example of how the data collected by the MGPSR receiver slowly fills in the transmit antenna pattern over time. The perspective is from the GPS transmit antenna frame showing the path of the receiving spacecraft over time. Plot (a) shows one day of data collection, and Plot (b) shows 35 days of data collection.

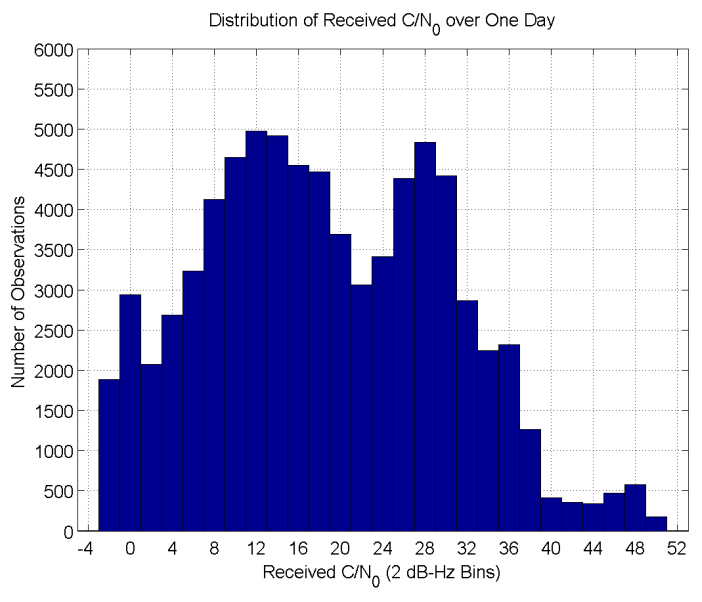

(a)

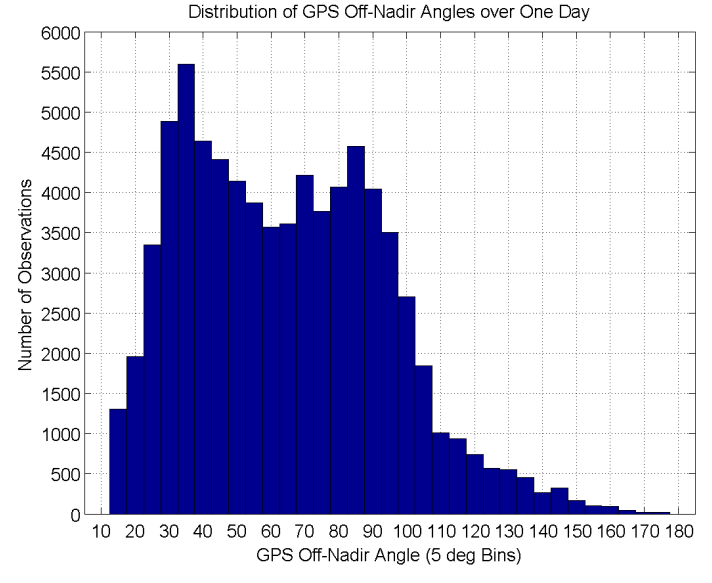

(b)

Figure 4: Histograms of the number of MGPSR observations taken over one day from a GEO spacecraft. Plot (a) shows the distribution of observations with respect to received carrier-to-noise ratio $\left(C / N_{0}\right)$ in $2 \mathrm{~dB}-\mathrm{Hz}$ bins. Plot (b) shows the distribution with respect to GPS off-nadir or off-boresight angle in 5 deg bins. 


\subsection{Reconstruction Approach and Tools}

The antenna gain pattern reconstruction process, shown in Figure 5, consists of two primary components: 1) an algorithm that uses the ODTBX software to reconstruct transmit gain from MGPSR receiver measurements and 2) a post-processing workflow that reconstructs a set of full patterns from a long-term batch of reconstructed measurements. Each of these major components, in turn, consists of the discrete steps described in this section.

The gain reconstruction process operates on $C / N_{0}$ measurements produced by the MGPSR receiver and uses ancillary data to resolve the receive/transmit geometry at each epoch, performs a link budget calculation to derive the GPS-transmitted gain value, and performs a number of quality checks at the measurement level. The MGPSR measurements are logged daily at 30-sec intervals for each visible satellite. The reconstruction algorithm operates on each measurement for a single PRN on a single day, resulting in transmit azimuth and elevation angles along with calculated transmit gain for each $C / N_{0}$ measurement. The individual processes in this component are:

1. Geometric modeling captures the problem geometry and calculates GPS transmit antenna-relative azimuth and elevation angles for each measurement;

2. Link budget modeling reconstructs the transmit antenna gain value from a received $C / N_{0}$ measurement; and

3. Pre-editing uses problem knowledge to detect and remove outlier measurements.

To process multiple dates and PRNs, an automated batch-processing system was developed to generate results across the full set of available data. A post-processing workflow was established to aggregate the generated data and produce the final set of products, primarily antenna gain patterns for each on-orbit GPS SV and averaged patterns for each GPS block. The post-processing is divided into distinct processes:

0. Aggregation collects PRN-specific data into SV-specific and block-average datasets,

1. Post-editing performs outlier detection and removal at the pattern level,

2. Binning transforms scattered measurements into a regular azimuth/elevation grid,

3. Filling uses interpolation to fill isolated missing bins,

4. Smoothing reduces noise in the final patterns, and

5. Normalization calibrates the final patterns against known independent sources (such as ground-measured data).

Each of these processes is discussed in detail in the following sections.

\subsection{Geometric Modeling}

The physical, geometric modeling of the GPS signal path involves calculating the angle of transmission from the perspective of the GPS transmit antenna, which results in a calculated transmit antenna azimuth and elevation angle associated with each measurement. Numerous data sources are needed for this process, including receiver and transmitter ephemeris, attitude, and antenna characteristics; general parameters such as leap seconds; and the MGPSR measurements themselves.

The MGPSR measurement data is available in two-hour batches for each individual PRN, at a 30-second timestep. The available data consist of over thirty distinct parameters as output by MGPSR, but the reconstruction process uses four primary ones: epoch, PRN, $C / N_{0}$, and pseudorange. This data is validated at the time of ingest by removing duplicated data values, and again during the measurement editing process. This 30 -second timestep forms the fundamental timestep of the reconstruction process.

Several important details must be considered when mapping GPS ACE measurements back to the originating GPS satellite and transmit antenna geometry: GPS PRN to SVN mappings, SV block specific attitude models, and GPS constellation ephemeris. GPS transmit antenna designs have changed with each block, and in the case of Block IIR, even within a single block. Therefore, it is necessary to perform antenna pattern reconstruction relative to the physical GPS satellite vehicle number (SVN), rather than a given pseudorandom noise (PRN) spreading code, which may be reassigned. Because the MGPSR measurements are associated with the PRN, PRN-to-SVN mapping is done 


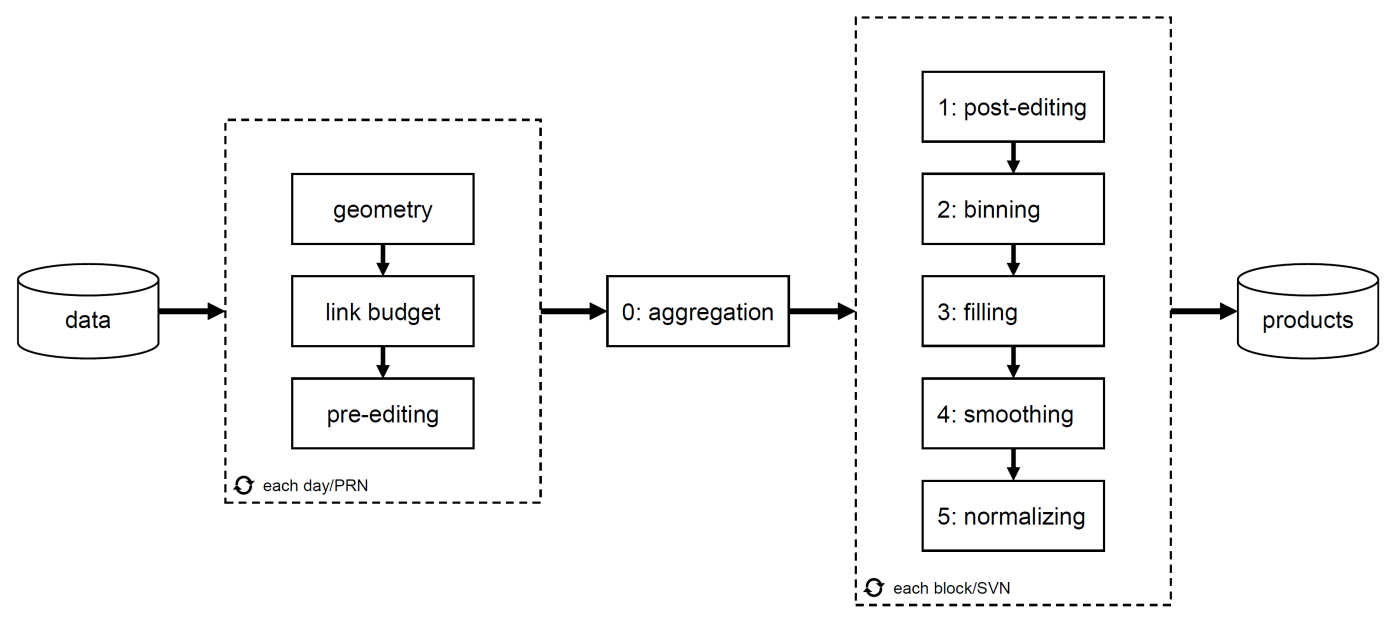

Figure 5: Gain pattern reconstruction process. Post-processing data levels $(0-5)$ are indicated on each post-processing step.

Table 1: GPS SVN/block mapping. Bold font indicates Block IIR satellites with the modernized antenna panel, processed as IIR-M SVNs.

\begin{tabular}{|l|l|}
\hline Block & Tracked SVNs \\
\hline IIA & $23,26,33-35,38-40$ \\
IIR & $41,43-46,51,54,56$ \\
IIR-M & $\mathbf{4 7}, 48,50,52,53,55,57,58, \mathbf{5 9 - 6 1}$ \\
IIF & $62-73$ \\
\hline
\end{tabular}

using a compiled history of PRN to SVN assignments. There are several sources for this data, including public files published by Analytical Graphics, Inc. (AGI) and the International GNSS Service (IGS). For this effort, an internal mapping dataset available within NASA and the USAF was utilized for convenience and validated against the public data to ensure agreement.

Each SVN was then mapped to a block type using publicly-available data. The block assignments used for this analysis differ from other sources because of the fact that four Block IIR satellites were configured with modernized antenna panels identical to those used on Block IIR-M vehicles. These were considered as part of Block IIR-M for the purposes of this analysis. Table 1 shows the SVN-block mapping used in this analysis, where the modernized IIR satellites are indicated with bold.

ODTBX computed the GPS constellation ephemeris from publicly-available GPS YUMA almanac data. Employing the almanac published on the day of a particular batch of MGPSR observations put the measurement epoch near the center of the almanac validity period. Standard algorithms were used to calculate GPS vehicle position at a given epoch from the almanac. Some consideration was given to using broadcast ephemeris data or post-processed ephemeris data available from external sources, but the error introduced via use of almanac data was considered negligible, as described in Section 6.3. By comparing one day of YUMA propagated almanac data with NGA precise SP3-format ephemerides for all PRNs, the maximum difference was found to be less than $4.2 \mathrm{~km}$, less than $4 \%$ of the magnitude necessary to cause a threshold error of $0.5 \mathrm{deg}$ or $0.1 \mathrm{~dB}$ in the resulting pattern.

For the transmitter attitude, the Bar-Sever model was implemented as described in [28]. This yaw model applies directly for the Boeing Block IIA and IIF satellites, in which the nominal attitude of the GPS satellites points the boresight of the GPS antenna at the center of the Earth and yaws about this axis in order to keep the solar panels perpendicular to the direction of the Sun. The nominal yaw angle is the angle of rotation about the $+Z$ body-fixed axis (nadir) from the velocity direction of the vehicle to the $+X$ body-fixed axis that ensures the $+X$ axis of the 
vehicle is pointing towards the Sun. The nominal yaw for the Lockheed Martin Block IIR and IIR-M satellites is oriented differently with the $-X$ axis pointed towards the Sun. Figure 6 shows this geometry for the yaw angle calculation for the various satellite blocks. The GPS satellites do not follow this nominal attitude behavior during a period of eclipse (the midnight turn) or when the nominal yaw rate would be too high at orbit noon (the noon turn). Because the behavior of the GPS satellites is not well defined during these periods, noon and midnight turn data are not included in the antenna pattern reconstruction process.

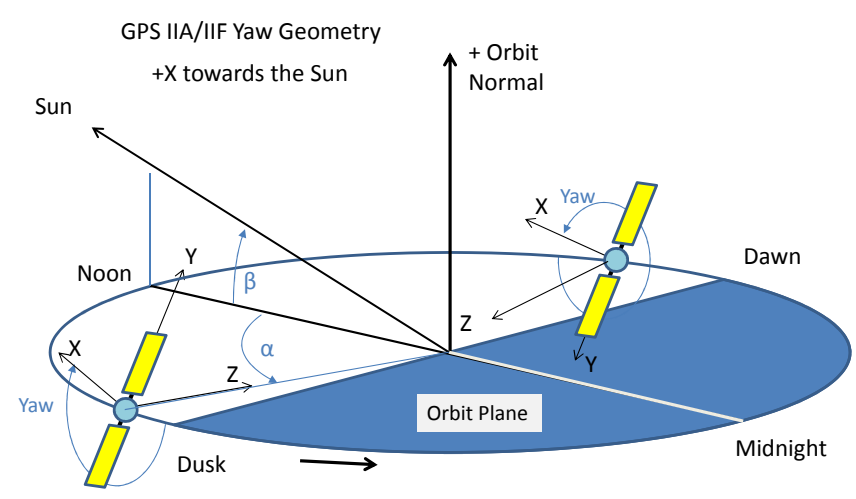

(a)

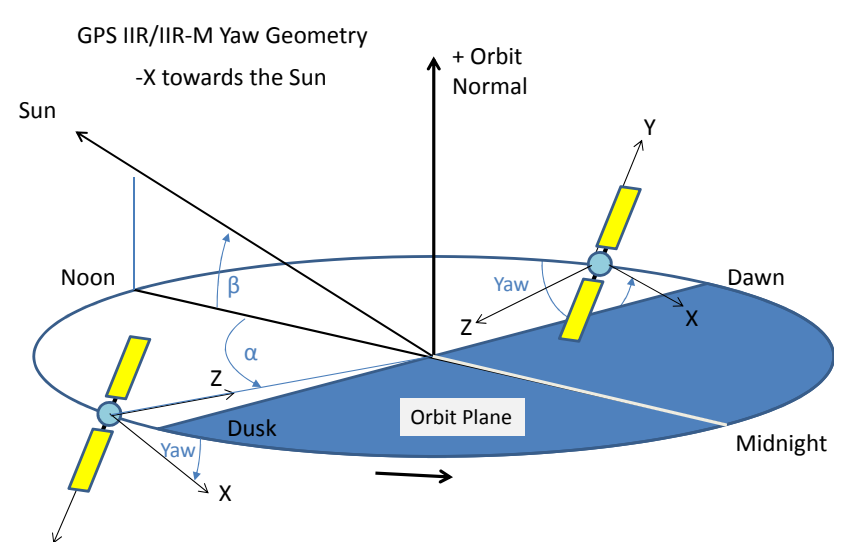

(b)

Figure 6: Geometry of the GPS transmitter yaw model for Block IIA and IIF (a) and Block IIR and IIR-M (b). The yaw angle is defined as the rotation about the nadir pointed $+Z$ body-fixed axis from the velocity vector direction in the orbital plane to the $+X$ body-fixed axis.

For the receiving spacecraft, orbit position is available via ephemerides provided at a 5-minute timestep in the Earth-centered, Earth-fixed frame. This data is first checked for validity and consistency, then is converted to the Earth-centered internal (J2000) frame and interpolated to the measurement times using divided difference Hermite interpolation. The FK5 reduction from Earth-fixed to inertial frames was performed using the IAU1980 nutation and precession model, and with a fixed polar motion model. Validity checks include expected timespan, expected orbit parameters, data discontinuities, invalid values, and large-scale visual spot-checks. All potential violations were logged as part of the automation process, investigated, and resolved via acceptance or exclusion of the data.

The spacecraft attitude is provided via a variable-step Euler angle ephemeris at approximately 5-minute intervals. The provided angles relative to the body frame are converted to quaternion form, "unwrapped" to resolve sign discontinuities, and interpolated to the spacecraft ephemeris times via spherical linear (slerp) interpolation. The resulting body-frame attitude is then rotated through a series of documented, fixed rotations to the inertial frame and interpolated again by the same method to the measurement times. The resulting rotation is used to determine receiver antenna pointing and yaw rotation about the boresight. Like the orbit ephemeris, the attitude is verified for validity and consistency by testing against expected timespans, expected pointing constraints, data discontinuities, invalid values and malformed attitude representations, and via visual spot-checks.

\subsection{Link Budget}

The effective isotropic radiated power (EIRP) of the GPS satellites cannot be directly measured with the GPS ACE data. Rather, it can only be inferred through carrier to noise density $C / N_{0}$ measurements, which include inherent modeling assumptions regarding system noise and other factors in the link budget. With knowledge of the received power $R_{p}$, the receiver antenna gain $A_{r}$, space-loss attenuation $A_{d}$, and the transmit power of the GPS satellite $P_{s v}$, the gain at the transmit antenna $A_{t}$ can be found. However, because the true transmit power of the GPS satellites is unknown and because there is modeling uncertainty in other aspects of the link budget, this method cannot fully separate the transmit power and the gain from the EIRP. For this reason, a nominal transmit power value was chosen for each block of GPS satellites as specified in Table 2. These transmit values meet the minimum required GPS 
power levels at the edge of the Earth and account for estimated excess power above spec based on receiver tracking data and knowledge of the main lobe patterns.

The final reconstructed antenna gain patterns from the GPS ACE measurements are normalized with respect to the ground measured data available for each of the GPS blocks. A more detailed discussion of the normalization method is presented in 4.10 .

The fundamental measurement used for the antenna pattern reconstruction is the GPS receiver's estimated carrierto-noise density ratio, $C / N_{0}$. In order to calculate the received power $R_{p}$, the noise of the system must be calculated. The effective antenna noise temperature is found by considering the thermal radiation from the Earth and the cosmic background. In general, the antenna noise temperature $T_{a}$ is given by:

$$
T_{a}=\frac{1}{4 \pi} \int_{4 \pi} G(\theta, \phi) T(\theta, \phi) d \Omega
$$

where $G(\theta, \phi)$ is the antenna gain and $T(\theta, \phi)$ is the noise temperature as functions of the antenna azimuth angle $\theta$ and elevation angle $\phi$. When integrating the temperature over the unit sphere, the Earth temperature was assumed to be $290 \mathrm{~K}$, and the cosmic background radiation was assumed to be $29 \mathrm{~K}$ at the GPS frequencies [29]. The receive antenna gain pattern was modeled as a second order polynomial which is uniform in azimuth. The thermal noise contributions from the Sun and Moon, when they were in the field of view, were transient and did not have a large impact on the average antenna noise temperature and, therefore, were not used.

The noise temperature of the front-end antenna electronics $T_{g}$, which include filters and low noise amplifier (LNA), can be found via the Friis Formula [30], assuming a fixed spacecraft temperature of $290 \mathrm{~K}$ and a fixed noise figure, $N F_{l}$, as:

$$
T_{g}=290\left(10^{\frac{N F_{l}}{10}}-1\right)
$$

It is also assumed that the LNA has a fixed gain, $G(\mathrm{~dB})$, and that there is a fixed cable loss, $L(\mathrm{~dB})$, between the front-end antenna electronics and the downconverter. The downconverter noise temperature $T_{d}$ is a function of the noise figure of the downconverter, $N F_{d}(\mathrm{~dB})$, and the total linear gain before the downconverter, $g=10^{(G+L) / 10}$ :

$$
T_{d}=290\left(\frac{10^{\frac{N F_{d}}{10}}-1}{g}\right) .
$$

Finally, the system noise temperature $T_{s}$ is simply:

$$
T_{s}=T_{a}+T_{g}+T_{d}
$$

Other losses accounted for include the receive antenna polarization loss (before the LNA), $A_{s}$, and receiver loss, $L_{r}$, which includes the increase in the noise floor due to the presence of multiple GPS signals in the L1 band and the analog to digital conversion loss on the ground.

The GPS signal carrier power at the receiving spacecraft just before the antenna is a function of the noise temperature of the receiver $N_{0}=10 \log _{10}\left(k T_{s}\right)$, where $k=1.38 \times 10^{-23} \mathrm{~W} / \mathrm{K}-\mathrm{Hz}$ is Boltzmann's constant, the measured carrierto-noise density ratio $C / N_{0}$, the receiver antenna gain $A_{r}$, and the contributions from other losses, $A_{s}$ and $L_{r}$. This relationship is shown in Eq. 9 where the losses are negative numbers $\left(A_{s}, L_{r}, N_{0}\right)$ and the gains are positive numbers:

$$
R_{p}=C / N_{0}+N_{0}-A_{r}+L_{r}+A_{s}
$$


The GPS signal carrier power at the receiving spacecraft just before the antenna can also be determined from the transmitter antenna gain $A_{t}$, the transmitter output signal power $P_{s v}(\mathrm{dBW})$, and the space-loss $A_{d}(\mathrm{~dB})$, which is a function of the distance between the transmitter and receiver and the link frequency L1:

$$
R_{p}=A_{t}+P_{s v}+A_{d}
$$

Since in this case, the transmit antenna gain is an unknown, the received power at the antenna calculated from the measured $C / N_{0}$ in Eq. 9 can be used to determine the transmitter gain by solving for $A_{t}$ in Eq. 10 as shown in Eq. 11:

$$
A_{t}(\theta, \phi)=C / N_{0}+N_{0}-A_{r}+L_{r}+A_{s}-A_{t}-P_{s v}
$$

A summary of the link budget parameters is presented in Table 2 and a more detailed look at the variation in the link budget parameters is presented in 6.2 .

Table 2: Constant link budget parameters assumed in the antenna gain pattern reconstruction.

\begin{tabular}{|l|c|c|c|}
\hline Link Parameter & Symbol & Value & Units \\
\hline Other Losses & $A_{s}+L_{r}$ & -1.31 & $\mathrm{~dB}$ \\
Noise Temperature & $T_{s}$ & 175.84 & $\mathrm{~K}$ \\
Block IIA TX Power & $P_{s v}$ & 14.90 & $\mathrm{dBW}$ \\
Block IIR TX Power & $P_{s v}$ & 15.00 & $\mathrm{dBW}$ \\
Block IIR-M TX Power & $P_{s v}$ & 14.30 & $\mathrm{dBW}$ \\
Block IIF TX Power & $P_{s v}$ & 14.30 & $\mathrm{dBW}$ \\
\hline
\end{tabular}

\subsection{Pre-Editing}

Before the data is aggregated for each GPS satellite, various data editing routines were applied to remove any erroneous data. The first edit check is for the GPS spacecraft yaw attitude. All the data during noon and midnight turns is removed because the behavior of the spacecraft is not well defined during those time periods. There are also periods of time in which the ground system configuration changed over the course of the multi-year data collection, which introduces intervals in which some of the MGPSR data is corrupted. To be conservative, all data recorded in the vicinity of configuration change periods was discarded. Finally, detailed analysis highlighted other periods of time when the MGPSR reported anomalous $C / N_{0}$ measurements attributed to anomalies with the receiver solution, or in some cases unexpected behavior of the GPS satellites. More details on these data editing methods and their verification is provided in Section 6.1.

\subsection{Aggregation}

The reconstruction process shown in Figure 5 generates a measurement transmit elevation angle, transmit azimuth angle, and calculated transmit gain value associated with each measurement for a single PRN on a single day. Utilizing the mappings discussed in 4.2, this data is then aggregated by GPS SVN and by block type to form SVN-specific and block-average patterns. The block-average patterns are generated by aggregating all measurements from SVNs within the block, prior to executing the post-processing steps. Table 3 summarizes the total number of measurements by block. Figure 7 shows the number of measurements per GPS SVN. SVNs 69-73 were the Block IIF satellites most recently added to the GPS constellation and, as such, were not available for GPS ACE data collection as long as some of the earlier satellites.

To generate a usable set of antenna patterns, a number of post-processing steps are implemented, as discussed in the following sections. As shown Figure 5, these steps are executed on each pattern individually. 
Table 3: Total number of measurements processed in each block. The IIR-M category includes IIR SVNs with the modernized antenna panel.

\begin{tabular}{|l|c|}
\hline Block & Total Measurements \\
\hline IIA & $04.8 \mathrm{M}$ \\
IIR & $19.1 \mathrm{M}$ \\
IIR-M* & $26.3 \mathrm{M}$ \\
IIF & $21.1 \mathrm{M}$ \\
\hline
\end{tabular}

Measurements by SVN

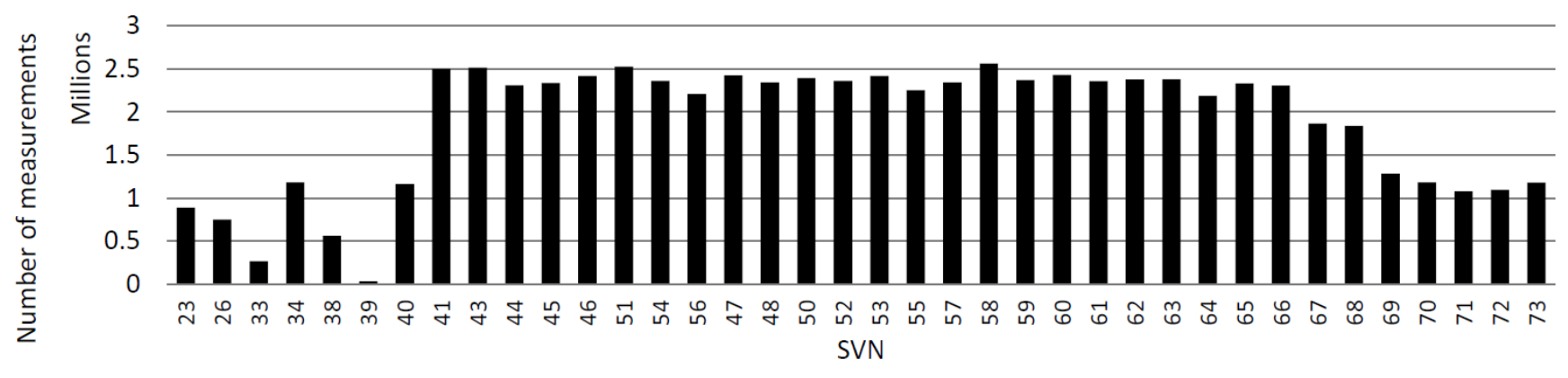

Figure 7: Quantity of measurements in dataset by GPS SVN. There are fewer measurements in the tails because the data set spans the period where Block IIA vehicles were being retired and Block IIF vehicles were being added.

\subsection{Post-Editing}

The measurement-specific pre-editing steps discussed above remove nearly all of the outliers prior to post-processing, but some remaining outliers become apparent after measurements are mapped to the transmit antenna coordinate frame. To address these remaining outliers, a sigma-editing step is used to remove outlier measurements in the context of the surrounding region of the pattern. The pattern is processed in uniform bins of 1 deg in azimuth and elevation angle. A sigma $(\sigma)$ value and mean is calculated for each bin, and all measurements outside of $3 \sigma$ from the mean are removed. This process is repeated in two passes, with the statistics recalculated after the first pass.

This editing step results in approximately $1 \%$ of measurements removed among the block-aggregated data.

\subsection{Binning}

The binning process interpolates scattered gain data onto a uniform azimuth/elevation grid for use as an input to mission analyses. There are many possible techniques that could be used for this process, with variables including the size of grid (the bins), the method for reducing the data within a bin, and the method for placement of the reduced bin value. In this case, the patterns were divided into a 1 deg azimuth/elevation grid with bin centers at integer deg values from 16 to $90 \mathrm{deg}$ off boresight across all azimuths, for a total of 27,000 bins. Data coordinate azimuth/elevation values were rounded for bin assignment, such that a value located at $18.3 \mathrm{deg}$ elevation and $54.5 \mathrm{deg}$ azimuth would be placed in the $18 \mathrm{deg} / 55 \mathrm{deg}$ bin. Within each bin, the mean is calculated of all gain values present in the bin, and the value is placed at the bin center. Some consideration was given to other values of bin size ( $0.25 \mathrm{deg}-2 \mathrm{deg})$ and reduction methods (mean, interpolation, nearest-neighbor, etc.). The adopted methods were chosen as the best combination of data volume, feature resolution, and simplicity.

\subsection{Filling}

Even with the measurement quantities shown in Figure 7, many SVN-specific patterns remain incomplete, with gaps in the grid varying from a few scattered empty bins, to large-scale incomplete features (particularly for some of the Block IIA SVs). Therefore, a step was implemented (when feasible) to interpolate into the gaps using data from the 
surrounding bins to form a filled pattern.

Recognizing that only small gaps will be recoverable, a threshold of $90 \%$ was established for pattern completeness - if the ratio of filled bins to empty bins in a given pattern was less than this threshold, gap-filling was not attempted. Once the threshold was met, interpolation was performed using a linear radial basis function interpolator as implemented by the SciPy Python package [31]. A trade was performed on interpolation methods, in which known portions of a complete pattern were removed and then filled in using a variety of available methods. This included cubic, quintic, Gaussian, and other varieties of the radial basis function method, and other interpolation functions provided by SciPy. The chosen linear method was selected among those available as the most accurate and applicable to the test data.

Figure 8 shows the SVN 62 pattern both before and after application of the gap-filling step.

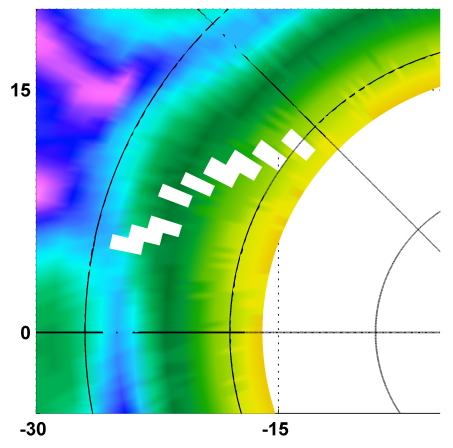

(a)

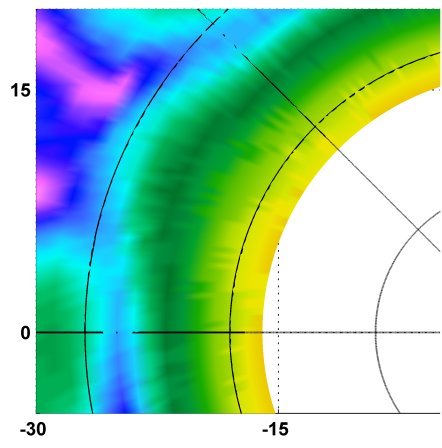

(b)

Figure 8: Example of linear radial basis function interpolation used to fill gaps in SVN 62 pattern. Image (a) features a series of 1x1deg gaps due to missing measurements in those bins. Image (b) shows the interpolated pattern.

\subsection{Smoothing}

The binned and filled data is an average of a large number of individual points in each individual bin, which does not create a smooth antenna profile, especially where the data was sparse or missing. It was also found that the data in the main lobe is noisier than the rest of the antenna pattern. This is assumed to be attributed primarily to the steep slope of the gain pattern in this region in conjunction with the long (30 second) integration period of the MGPSR. A secondary contributor to noisier main lobe signals could be perturbations induced as main lobe signals pass closely by the limb of the Earth. However, only GPS signals with a transmit off-boresight angle of 16 deg or greater (corresponding to a height of ray-path (HORP) altitude of approximately $1200 \mathrm{~km}$ ) were included in post-processing. To mitigate bin-to-bin measurement noise, a two-dimensional Gaussian lowpass filter was applied to the individual patterns after the filling step. Replicating the border elements at the edge of the pattern for the filter was not a sufficient approach in the main lobe in the off-boresight dimension again because of the steep slope. Therefore, a two tiered filter window was applied to the pattern: a 1x7 deg size rectangle in the main lobe from 16 to $30 \mathrm{deg}$ and a $3 \times 7 \mathrm{deg}$ for the rest of the pattern. The end result is a much smoother pattern that can be used to

compare against ground measured data. Figure 9 shows the azimuthal variation at 18 deg off-boresight in a block IIR antenna pattern and the output of the smoothing process. 


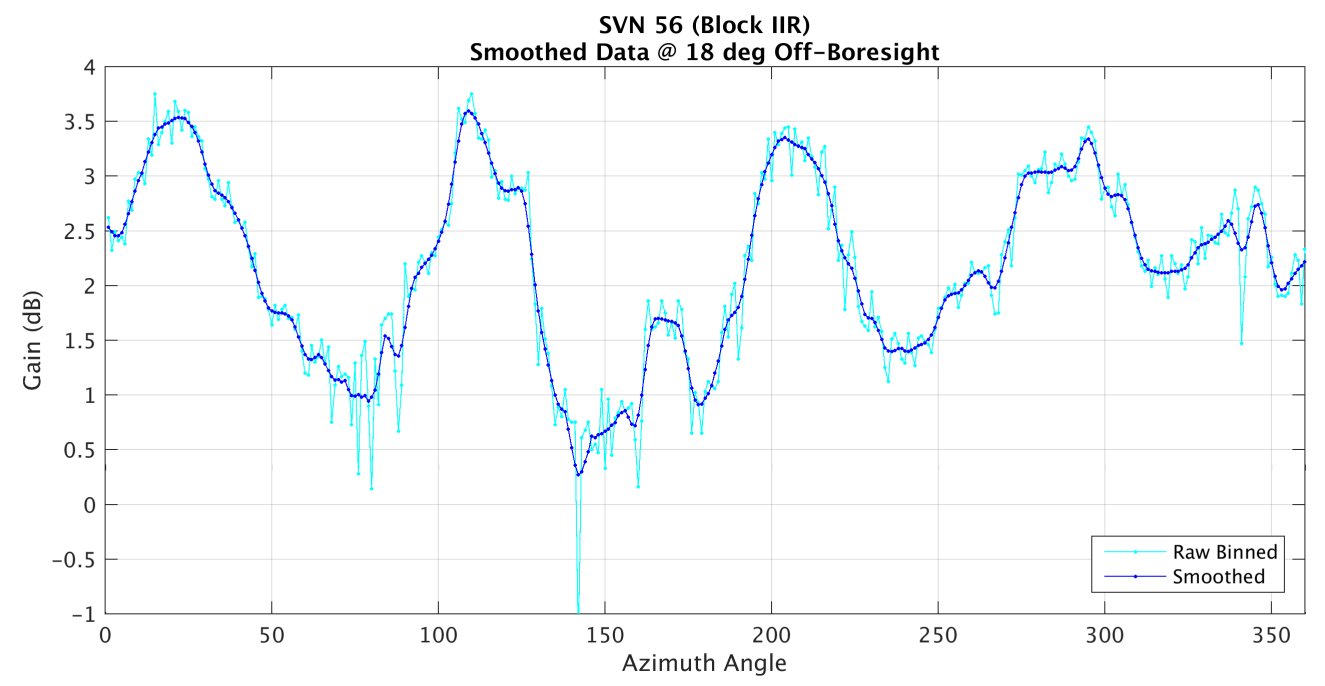

Figure 9: Example data from a block IIR (SV 56) satellite. The binned data is smoothed with a Gaussian lowpass filter.

\subsection{Normalizing}

As mentioned previously, the true transmit power of the GPS satellites is not publicly available information and therefore there exists an unknown parameter in the link budget which makes it difficult to determine the absolute isotropic gain for the reconstructed antenna patterns. However, the L1 transmit directivity and gain patterns for each individual Block IIR and IIR-M satellite are publicly available and therefore can serve as an anchor point for the reconstructed patterns. Additionally, the Aerospace and NASA GPS ACE team members have access to Block IIF gain patterns of the main lobe response at L1 that to-date have not been released to the public. The final step in the antenna pattern reconstruction process uses these data sets to normalize the gain patterns to these reference data sets, thereby producing a final product that should closely represent the absolute gain of the GPS antenna panels, separate from the transmit power.

The normalization method involves taking multiple elevation cuts from 16 to 23 deg off-boresight in the main lobe at $1 \mathrm{deg}$ steps in azimuth and then finding the least squares best fit of the smoothed data to the ground measured data. The average of the best fit offsets is then calculated which results in one absolute offset number per SV. A summary of the mean offset per block, which represent a combination of the difference between the approximate transmit power and the true power and the other uncertainties in the link budget, are presented in Table 4 . These offsets are then applied globally to the per SV patterns to produce the final reconstructed antenna gain products. For the Block IIA patterns, measured antenna pattern data was not available to reference, so the IIF data was used in its place due to their similarities. For the block-average patterns, the final individual normalized patterns were averaged together bin-by-bin to produce the final products.

Table 4: Mean global offsets per block used to normalize the final reconstructed antenna patterns. Note: Block IIA SVS 33, 35, and 39 are not included in this list because they were taken out of the constellation before enough data could be recorded.

\begin{tabular}{|c|l|c|}
\hline Block & SVNs & Mean Global Offset (dB) \\
\hline IIA & $23,26,34,38,40$ & 5.6 \\
IIR & $41,43-46,51,54,56$ & 5.3 \\
IIR-M & $47,48,50,52,53,55,57-61$ & 4.0 \\
IIF & $62-73$ & 4.6 \\
\hline
\end{tabular}




\subsection{Products}

The primary final product generated at the end of the reconstruction process is a set of antenna gain patterns on a regular $1 \mathrm{deg}$ azimuth/elevation grid, extending from 16-90 deg in elevation. These patterns are generated for each individual SVN for which data is available, and for each block as a whole using aggregated and averaged data.

A number of graphical and verification products are also generated for each pattern, at different levels of processing, from level 0 (raw aggregated gain measurements) to level 5 (normalized final products). These include plots of antenna gain in polar form, per-bin measurement counts, per-bin sigma values, and the familiar Cartesian plots of gain over elevation angle at regular azimuth cuts. These types of products were extremely valuable for verification, and a number of them are discussed in the following sections.

\section{Results}

\subsection{Reconstructed Transmit Antenna Patterns}

This section presents an overview of the final products generated from the post-processing of the GPS ACE measurements. Full results are planned to be released as supplementary data files. The first set of figures shown are heat maps representing block-average transmit gain measurements, created by averaging the gain patterns of the individual satellites within each block to create an average pattern for that block. These plots represent the final "level 5" products, incorporating all post-processing discussed in the previous section. GPS ACE measurements are plotted between 16 and 90 deg off transmitter boresight. The 16 deg off-boresight angle mask corresponding to a height of ray path (HORP) altitude of approximately $1200 \mathrm{~km}$; measurements below this mask were not processed due to perturbation from passing close to the limb of the Earth.

Figures 10 and 11 show side-by-side comparisons between the GPS ACE reconstructed patterns and ground-based gain pattern measurements [5] for Block IIR and IIR-M, respectively. It is immediately apparent that GPS ACE reconstructed antenna patterns demonstrate excellent agreement with the antenna patterns measured by Lockheed Martin before launch, with both gross and fine-scale features common to both data sets.

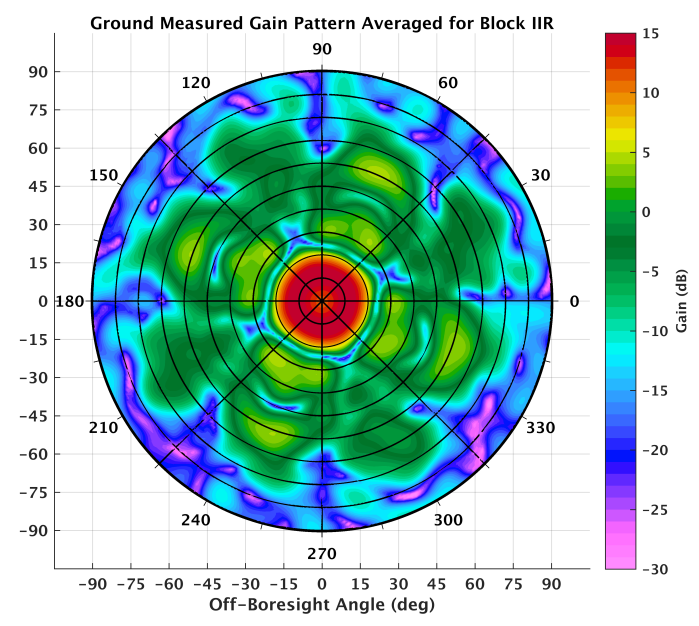

(a)

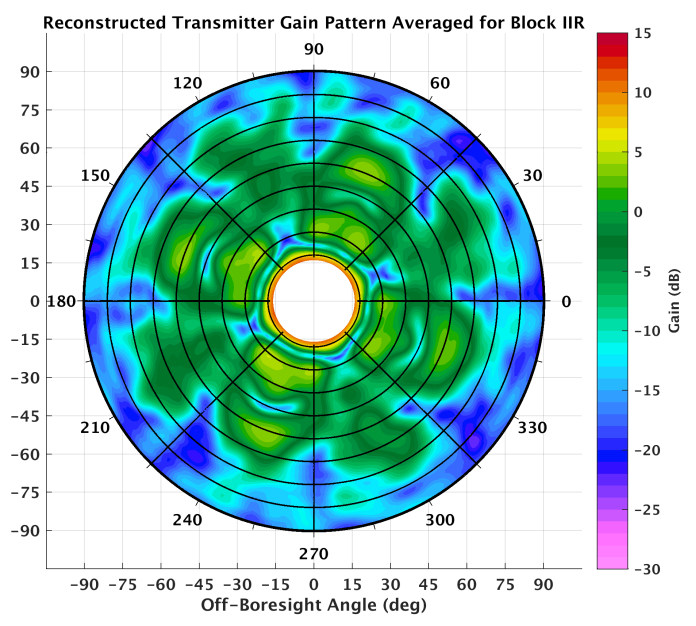

(b)

Figure 10: Comparison of Block IIR ground measured antenna pattern (a) [5] to reconstructed antenna pattern from GPS ACE flight data (b) as heat maps to 90 deg off boresight.

Two major differences are visually apparent between the ground-measured and reconstructed patterns. First, the reconstructed data only contains the outer edge of the main lobe signal, due to the reception geometry at GEO and the applied 16 deg off boresight angle mask. Second, the ground-measured patterns feature deeper antenna nulls than the reconstructed patterns. This is most apparent by comparing the outer edges of each plot, near 90 deg off 


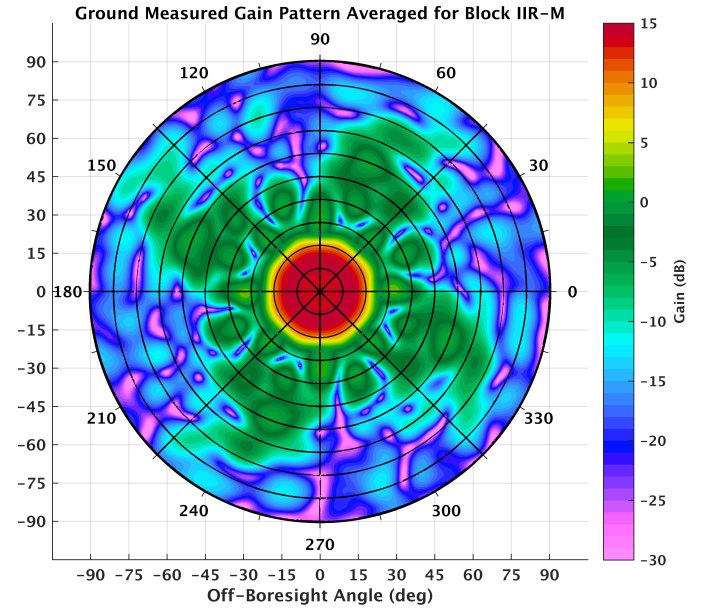

(a)

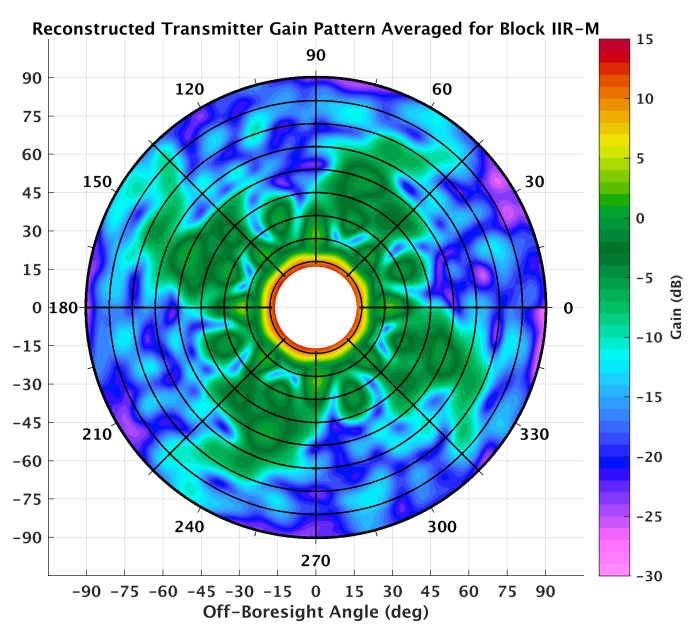

(b)

Figure 11: Comparison of Block IIR-M ground measured antenna pattern (a) [5] to reconstructed antenna pattern from GPS ACE flight data (b) as heat maps out to 90 deg off boresight.

boresight angle. In Figure 12, there is a deep null in the ground measured pattern that is not entirely captured in the reconstructed pattern. There are multiple potential causes. Review of one of these areas in the observation data identified measurements in the $20 \mathrm{~dB}$ deep null, but found that the null was captured at different epochs at slightly different angles, causing it to be smoothed out in the reconstruction. Other potential factors include limitations of the MGPSR receiver to track through the deepest nulls; the choice to use $1 \mathrm{deg}$ bin size, which results in a greater bin area near the edge of the pattern; and temporal variations in the null placement in the pattern itself.

It is important to note that the lack of deep nulls in the reconstructed data did not affect the normalization vs. ground-measured data, since this normalization was performed using elevation cuts from 16 to 23 deg off-boresight angle. Because most space users do not track through these outer null regions, this difference is not expected to result in optimism in future analyses using these results.

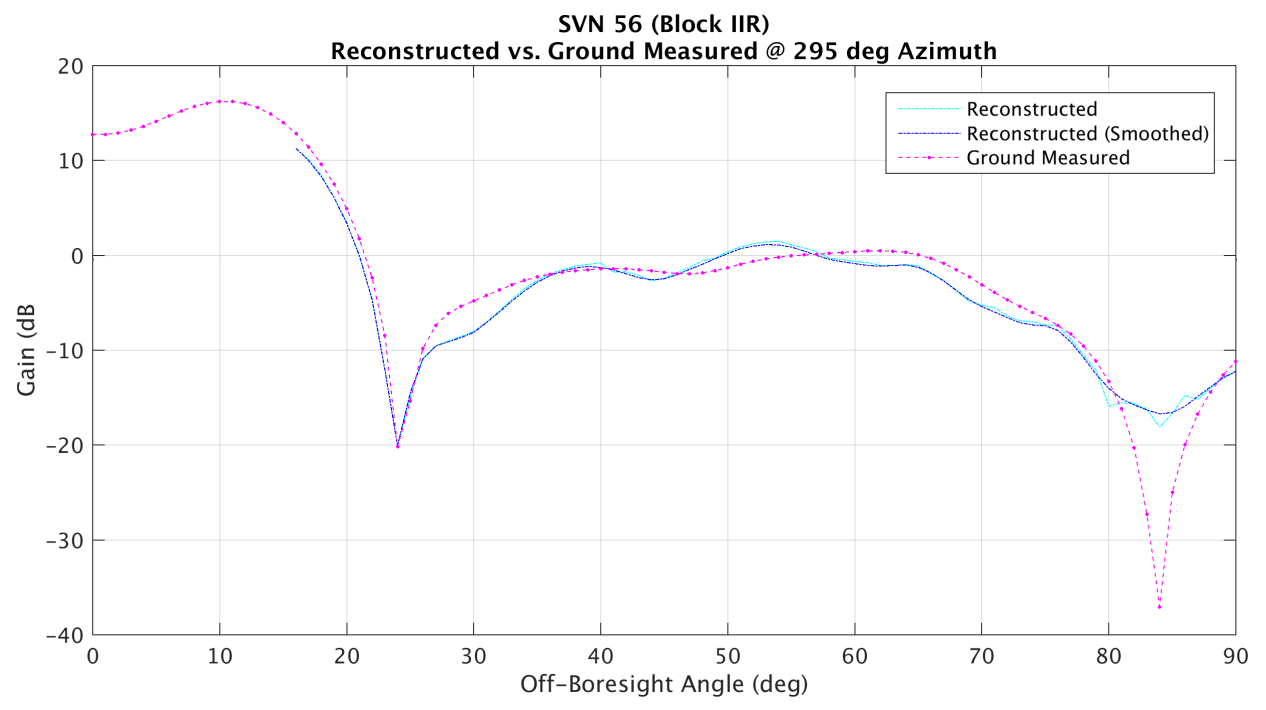

Figure 12: Example comparison of reconstructed antenna pattern vs. ground measured data from a Block IIR (SVN 56). This shows how the nulls in the reconstructed data are shallower than the ground measured pattern.

As can be seen in the following figures, sufficient GPS measurements were recorded from very weak GPS signals to 
completely reconstruct the full block-average patterns, and most individual SVN patterns, to 90 deg off-boresight. This data has previously only been available for Blocks IIR and IIR-M. As expected, the highest gain values are associated with main lobe region within approximately $23 \mathrm{deg}$ of the transmitter boresight. The highest side lobe signals generally have 10-15 dB lower gain than the peak main lobe gain. The IIR satellites have the highest peak side lobe gain of any of the GPS satellite blocks. Comparing IIR (Figure 10) and IIR-M (Figure 11) patterns, it is apparent that the modernized IIR-M antenna panel design suppressed the gain of the side lobe signals to optimize gain in the main lobe.

Figure 13 provides block-average reconstructed antenna patterns for GPS IIA and GPS IIF satellites. It is interesting to note that the Block IIF gain patterns are very similar to Block IIA, except that the pattern is clocked with a 45 deg relative offset. The similarities are expected, as both blocks were produced by Boeing. Also, no ground-measured data has been publicly released for the Block IIA satellites, and only limited data has been made available to the authors for Block IIF, so no direct comparison is presented here.

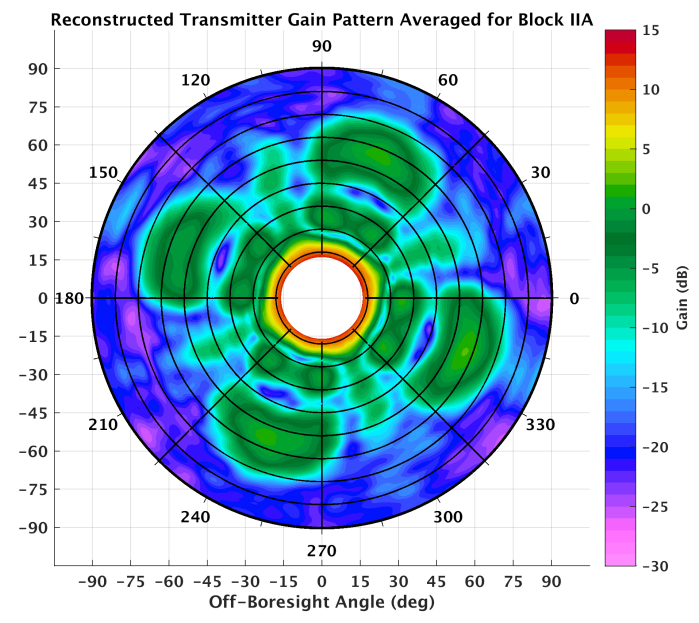

(a)

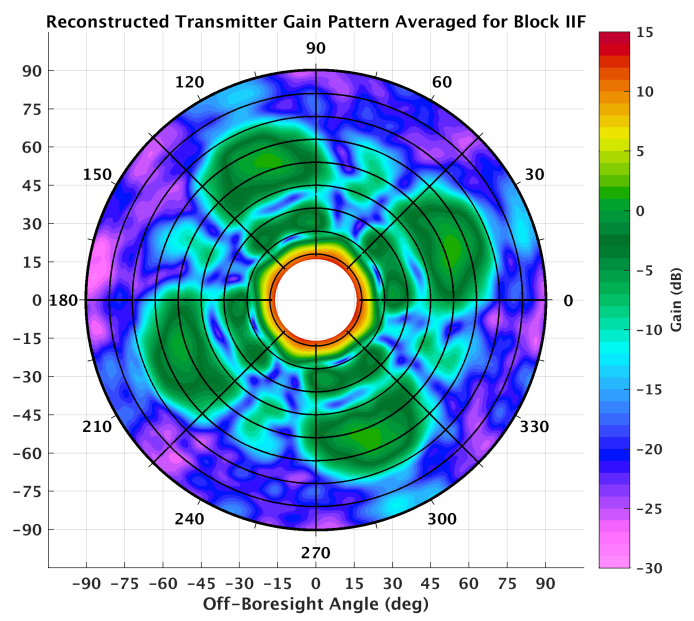

(b)

Figure 13: Comparison of Block IIA reconstructed antenna pattern (a) to Block IIF reconstructed antenna pattern (b) from GPS ACE flight data as heat maps out to 90 deg off boresight.

Figures 14 and 15 provide Cartesian plots of GPS ACE block-average gain versus elevation angle at regular azimuth cuts, illustrating more clearly the quantitative variation in gain across different azimuthal cuts.

In Figure 16 a comparison of the reconstructed pattern vs. the ground measured data for SVN 56 for an off-boresite cut at $30 \mathrm{deg}$ (first side lobe) shows that the detailed variations in azimuth are captured in the recostruction process. This provides confidence in the reconstruction process for the Block IIA and IIF data for which the ground measured data is not available in the side lobes. 


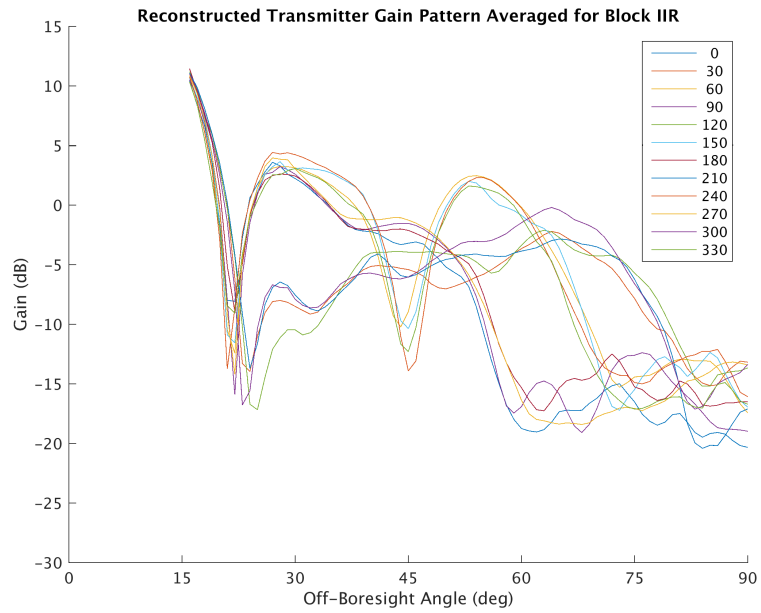

(a)

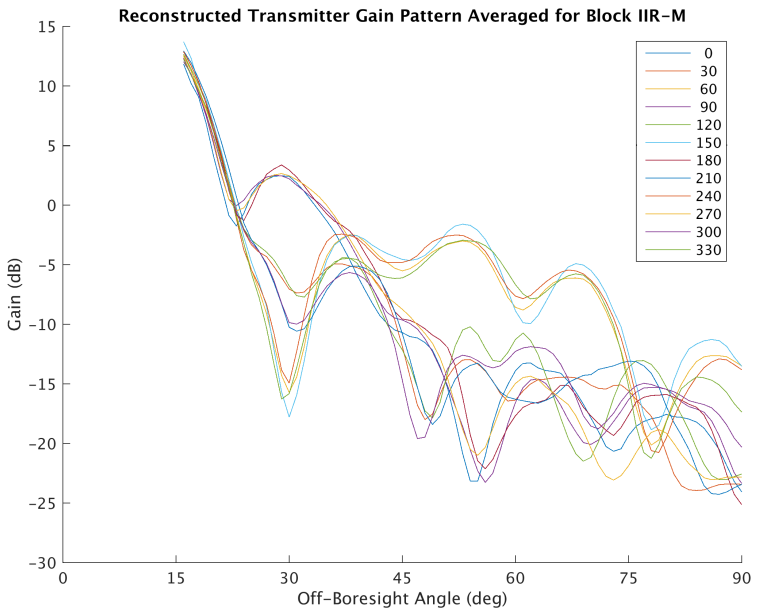

(b)

Figure 14: Comparison of Block IIR reconstructed antenna pattern (a) to Block IIR-M reconstructed antenna pattern (b) from GPS ACE flight data as azimuth cuts every $30 \mathrm{deg}$.

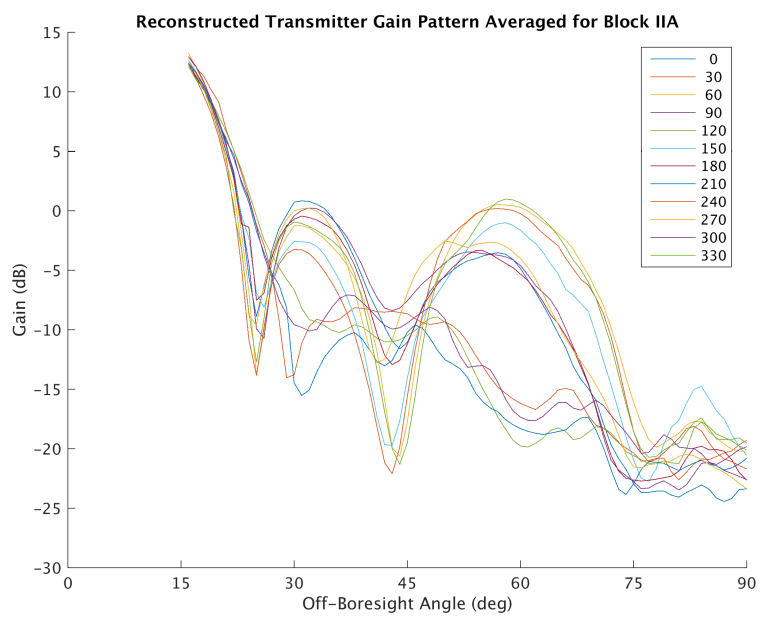

(a)

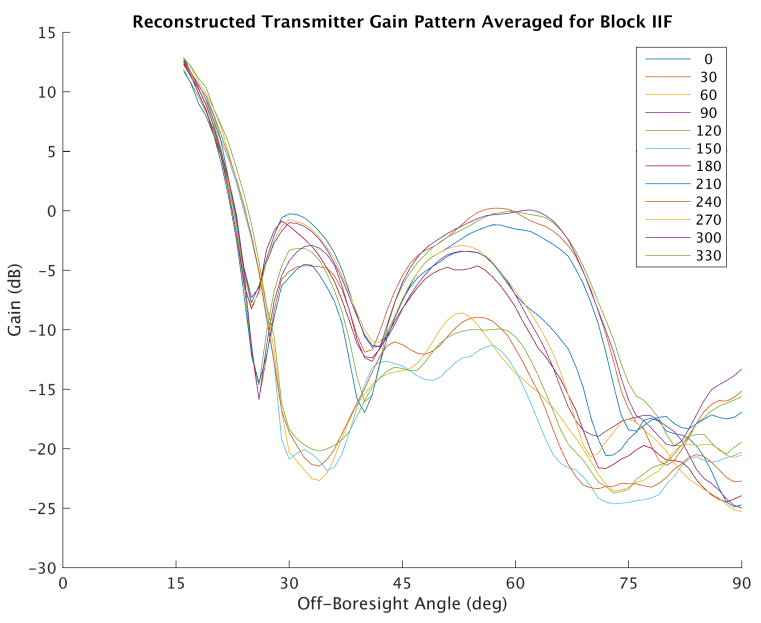

(b)

Figure 15: Comparison of Block IIA reconstructed antenna pattern (a) to Block IIF reconstructed antenna pattern (b) from GPS ACE flight data as azimuth cuts every $30 \mathrm{deg}$. 


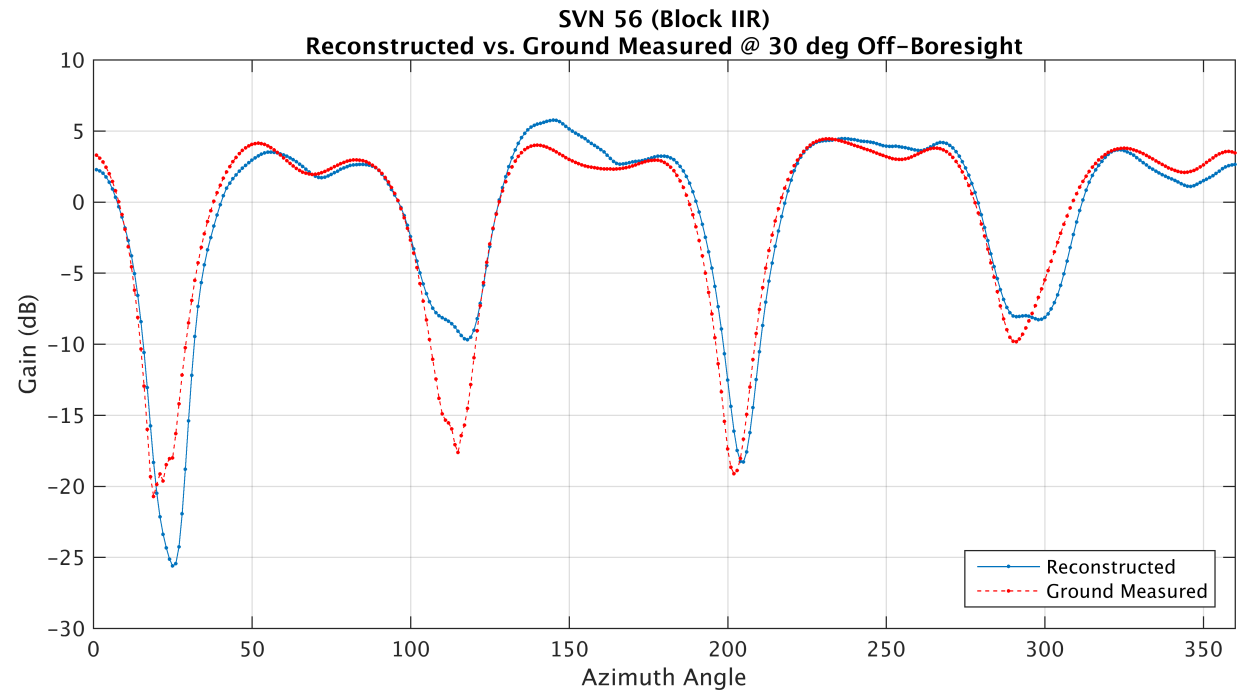

Figure 16: Example comparison of reconstructed antenna pattern vs. ground measured data from a Block IIR (SVN 56) using an off-boresight cut at $30 \mathrm{deg}$ (first side lobe). 


\subsection{Comparisons Between Block Average and Individual Gain Patterns}

Comparisons were made between the block-average antenna patterns and individual reconstructed patterns for each SV within the block.

This analysis demonstrates that variations in gain from SVN to SVN within a block are largely not significant. Figure 17 shows that the reconstructed block-average patterns closely match the individual reconstructed patterns to approximately $75 \mathrm{deg}$ off boresight, which indicates that there are no large variations in the majority of the antenna patterns between individual satellites within a block. These results indicate that for constellation modeling and analysis purposes, block-average patterns can generally be used in place of modeling the side lobe shape of each individual satellite.

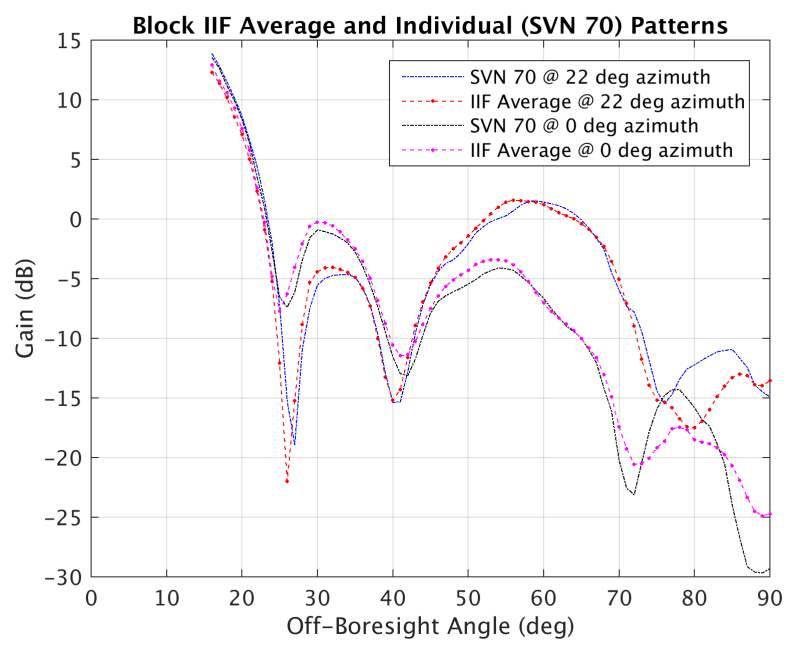

(a)

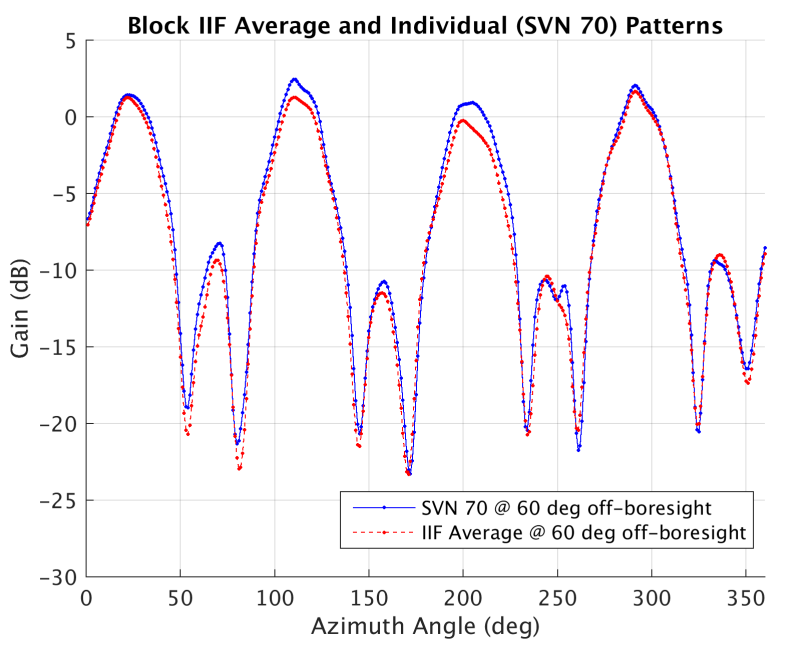

(b)

Figure 17: Comparison of the Block IIF average reconstructed pattern vs. an individual SVN 70 reconstructed pattern. Plot (a) shows two azimuth cuts at $22 \mathrm{deg}$ and 0 deg. Plot (b) shows the azimuth variation at 60 deg off-boresight (second side lobe).

However, it was found that there are mean differences in gain between patterns (before normalization). Because a fixed transmit power was applied uniformly to each block in the link budget, these differences represent both the uncertainty in the link budget calculations - which are discussed in greater detail in Section 6.2 - as well as any actual differences in transmit power between individual satellites within a block. Figure 18 shows the mean difference across the entire pattern for each GPS satellite vs. a reference vehicle in the block. The plot clearly shows that SVN 70 and 73 have increased measured gain compared to the other Block IIF SVNs. This may be an indication of higher transmit power on those satellites. 


\section{SV-SV mean differences within block \\ Nulls $<-20 \mathrm{~dB}$ ignored}

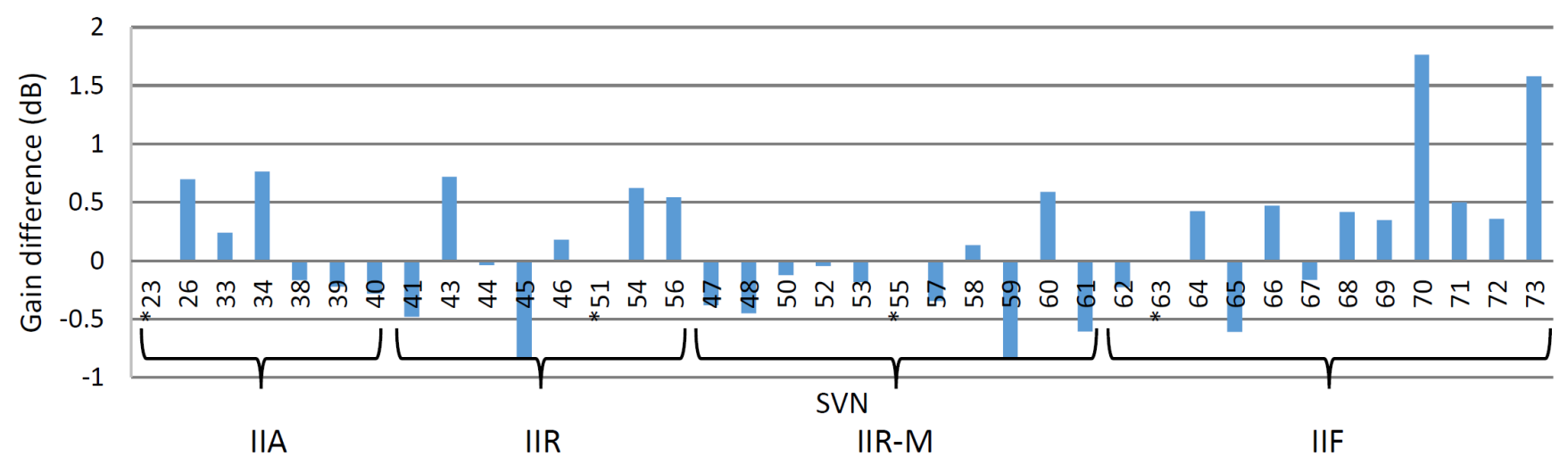

Figure 18: Mean gain differences between individual SVN patterns within each block, excluding deep (less than -20dB) nulls. SVNs used as references are marked with $(*)$. Block IIR-M includes IIR SVNs with the modernized antenna panel.

\section{Verification}

This section describes the analysis performed to verify the the reconstructed gain patterns and identify anomalous features in the data.

\subsection{Data Editing}

There are effects that cause errors and anomalies in the dataset. This subsection describes how that data was identified and removed from the full dataset.

\subsubsection{Spacecraft Attitude}

As mentioned previously, because the yaw behavior of the GPS satellites is not well defined during noon and midnight turns, the data collected during these periods are not included in the antenna pattern reconstruction process. In order to verify that the yaw model correctly identifies the period of time during which the spacecraft attitude is ill defined, spot checks were performed for SVNs 34 (Block IIA) and 61 (Block IIR). The received $C / N_{0}$ values were plotted for each SV for a 5 hour window during which an eclipse was predicted to occur and the receiver was tracking a strong side lobe signal. The GPS satellite attitude maneuver was clearly identifiable during both the shadow and post-shadow period as a variation in received signal power with fluctuations of up to $25 \mathrm{~dB}$ as the spacecraft presented a series of large nulls in the side lobes. As an example, Fig. 19 shows a midnight turn of SVN 34. This check verified that all data points received during these attitude maneuvers are properly discarded by the yaw edit logic before generating the reconstructed antenna patterns. 


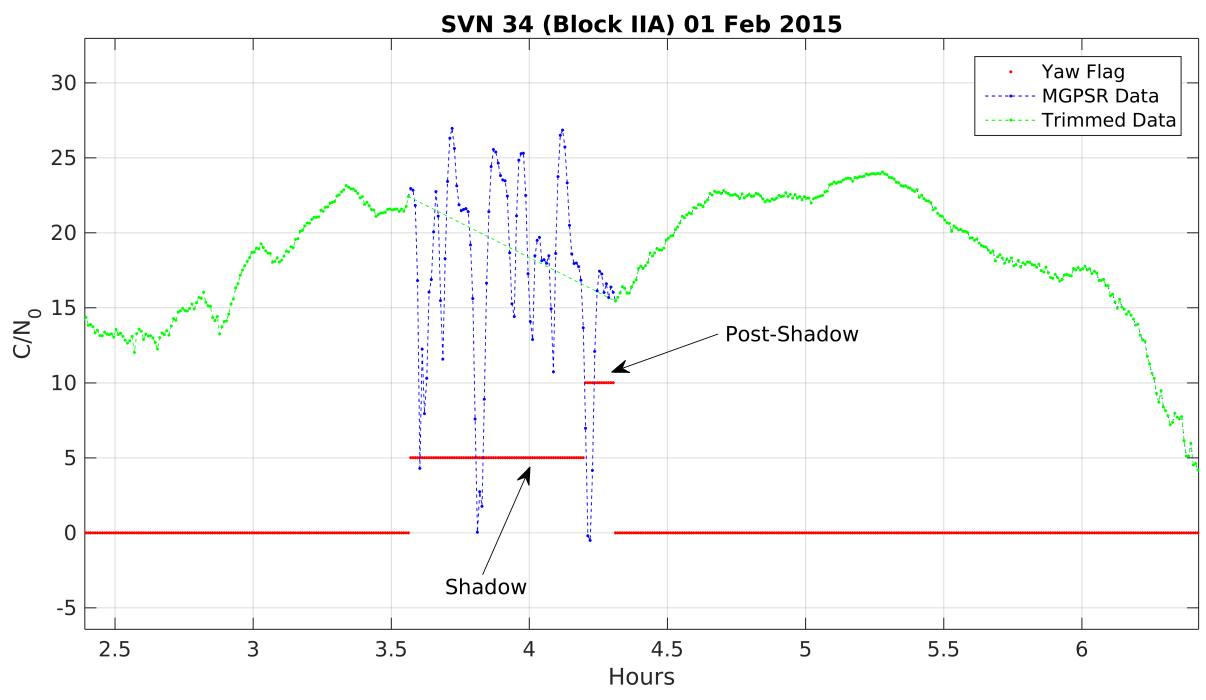

Figure 19: SVN 34 Block IIA Received $C / N_{0}$ over a midnight turn (eclipse).

\subsubsection{Ground Configuration Changes}

The MGPSR receiver has been nearly continuously processing the digitized GPS signals for several years. Inevitably, parts of the ground system upstream of the RF digitizer are occasionally brought off-line or have undergone some other configuration changes. These configuration changes could introduce errors in the dataset when they occur. The dates and times of these changes are recorded and were available to edit out bad data. Because the MGPSR processes the digitized signal in two hour chunks, when a change occurs, the two hour data set is corrupted. The MGPSR then automatically adjusts to match the current ground system configuration for the next two hour period. Spot checks were performed for every type of configuration change experienced over the data collection period. These checks consisted of plotting the received $C / N_{0}$ during each event and inspecting the quality of the data during those time periods such as looking for outliers or non-physical behaviors. Fig. 20 is an example of one such spot check. It was found that the time stamps associated with configuration change logs were only accurate to within approximately 5 minutes of the actual start and stop times as observed in the plots. Therefore, all data 15 minutes before a recorded configuration change is discarded before generating the reconstructed antenna patterns. The 15 minutes was verified to be sufficient margin by spot checking several configuration changes. 


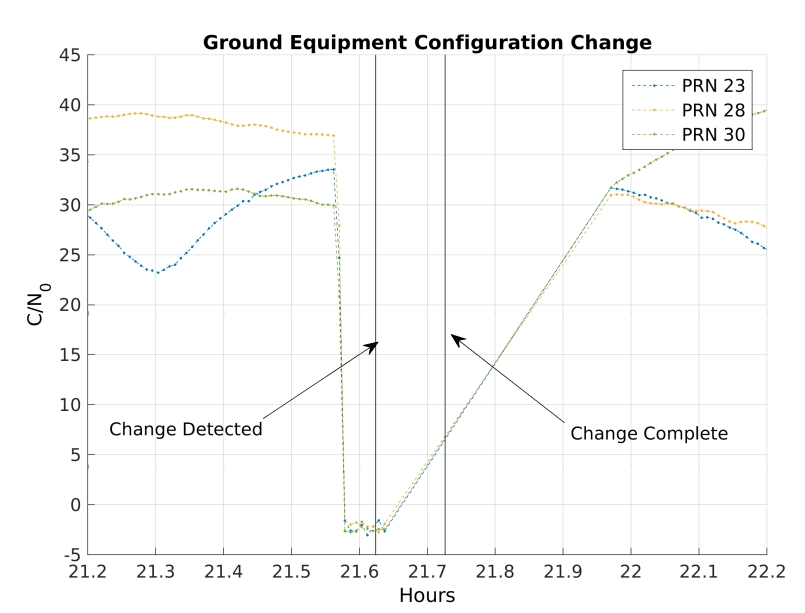

(a)

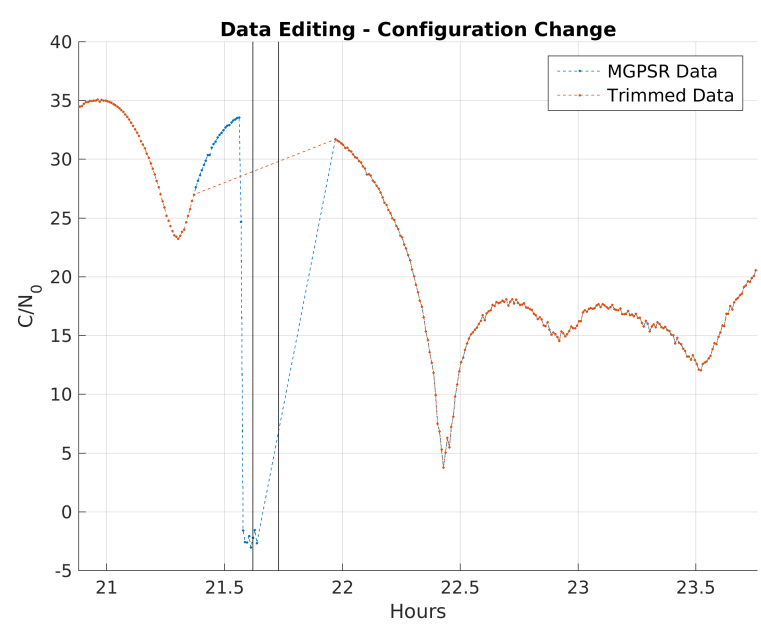

(b)

Figure 20: Received $C / N_{0}$ over a ground equipment configuration change. Plot(a) shows a sample of the signal drop for three PRNs. Plot (b) shows the pre and post-edit data sets for one PRN. A conservative margin was used. In this particular case, data was masked 15 minutes before the stated time of the configuration change until the end of the 2 hour arc.

\subsubsection{Anomalous Tracking Data}

From one day to the next, the $C / N_{0}$ profile as a function of time for a particular GPS SV does not change significantly because of the unique geometry between the GEO receiver and the MEO GPS transmitters. That repeatability can be exploited to do additional data integrity spot checks on the received $C / N_{0}$ data. Occasionally, the MGPSR receiver will have a bad tracking pass for 2-4 hours. The root cause is not always known, however, the possibilities include cross-correlations and unplanned GPS spacecraft activities such as off-nominal spacecraft yaw attitudes. Examination of plot (a) in Fig. 21 shows one such example where the tracking pass on one day appears to represent an off-nominal behavior of the SV and the next day the pass looks clean. It was confirmed by the GPS operators that on this particular day for SVN 62, this behavior was caused by a 20 deg yaw bias applied to the spacecraft around local dusk. Since it would not be possible to catch all such events manually, an automated process for detecting and removing this data was developed. In addition to the $C / N_{0}$ and pseudorange measurements reported at the end of each two hour data processing batch, the MGSPR outputs information on the pseudorange measurement error standard deviations, $\sigma_{m}$ and estimated ionospheric delay correction, $d_{i}$, both of which can be used to find outliers. If $\sigma_{m}$ has a large jump while the receiver is tracking strong signals $\left(C / N_{0}>25 \mathrm{~dB}-\mathrm{Hz}\right)$, it is an indication that there may be bad data. Additionally, if the receiver reports very weak signals $\left(C / N_{0}<5 \mathrm{~dB}-\mathrm{Hz}\right)$ for a long period of time when the $d_{i}$ correction flag is low, i.e., the signal is not being attenuated by the atmosphere, then the data is also flagged as anomalous. Plot (b) in Fig. 21 shows the same Day 1 with the bad pass in the left plot for a longer 8 hour arc and demonstrates how the automated process was able to detect and flag the data that needed to be discarded. It should be noted that while the $d_{i}$ and $\sigma_{m}$ flags could also be used to remove the main lobe data that is attenuated by the atmosphere, it was decided that this data should be removed after the final antenna pattern reconstruction using an angle off-boresight mask corresponding to the height of the Earth's ionosphere as seen from GEO, rather than in the pre-processing.

To aid in identification of outliers, a custom tool called PatternExplorer was developed for interactive interrogation of the final antenna pattern products. This tool allows the user to load a product, usually a level-0 gain plot, interactively focus on specific features of the pattern, select individual bins of measurements, see measurement statistics and distribution details, and perform per-bin sigma-editing experiments. It also enables interactive interrogation of specific measurements back to the source data for investigation of outliers and other features. The PatternExplorer interface is shown in Fig. 22. 


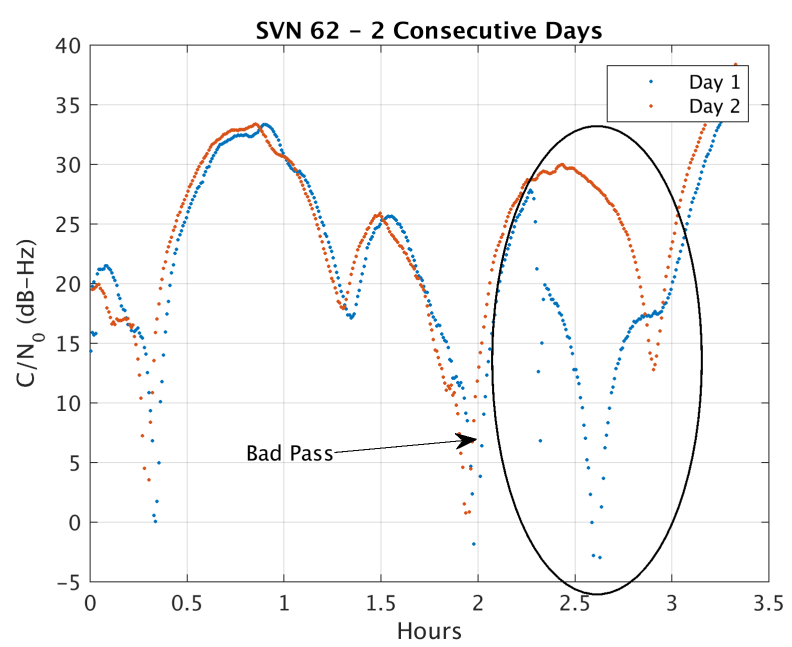

(a)

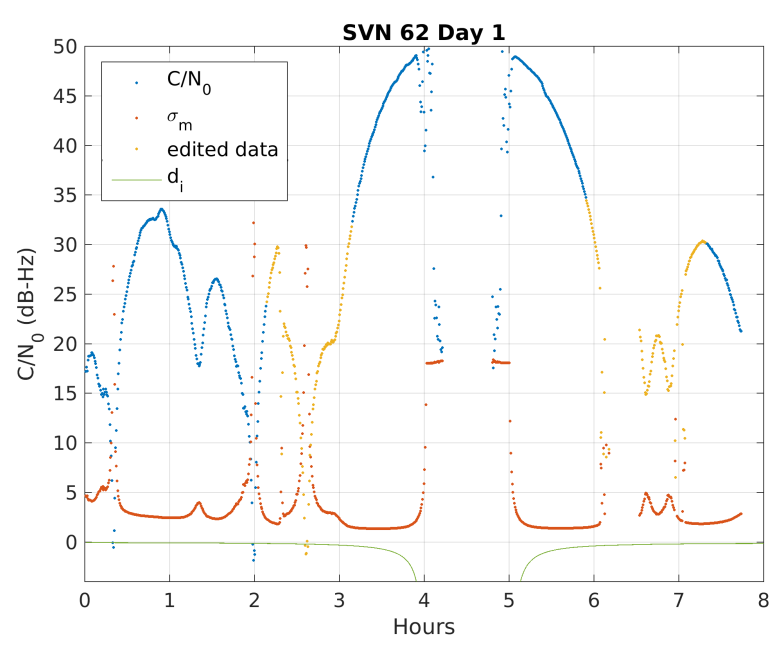

(b)

Figure 21: Plot (a): Received $C / N_{0}$ on two consecutive days for SVN 62 where the Day 1 data appear to have a discontinuity at around 2.25 hours into the day caused by a yaw maneuver. The Day 2 data look nominal.

Plot (b): Received $C / N_{0}$ for an 8 hour arc on Day 1 for SVN 62. The automated data editing process found the bad pass at hour 2.25 and another bad pass starting at hour 5.75.

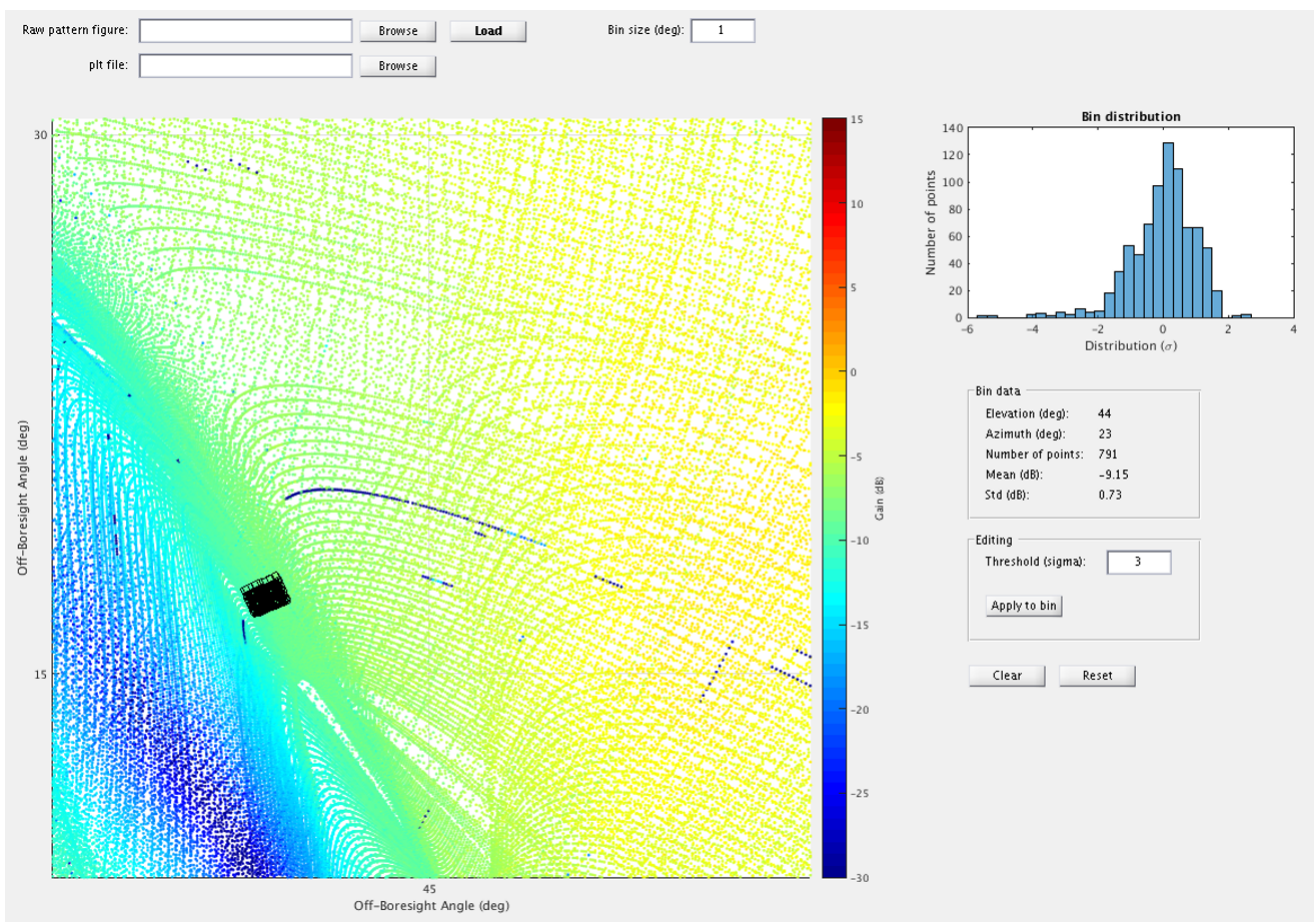

Figure 22: The interface of the PatternExplorer tool, showing interrogation of an individual bin of raw unedited measurements (black dots in lower left of pattern) and associated statistics. 


\subsection{Link Budget Summary}

There are several sources of error in the GPS ACE antenna pattern reconstruction link budget. As described in Section 4.3 and shown in Eq. 11, the measured carrier to noise density ratio $C / N_{0}$, noise temperature of the system, $N_{0}$, and the receive antenna gain, $A_{r}$, are required to determine the transmitter gain. These are the largest sources of error in the link budget and are discussed in greater detail in the following sections.

\subsubsection{Uncertainty in Carrier to Noise Density}

The MGPSR algorithm for estimating the carrier to noise density ratio was presented in Section 3.2.2. One method of determining the uncertainty in the MGPSR $C / N_{0}$ calculation is to compare the MGPSR measurements to the Navigator GPS receiver outputs for the same pass. Since the Navigator is not as sensitive as the MGPSR, a main lobe pass was chosen for this analysis. The Navigator $C / N_{0}$ estimator is a measurement of the ratio of narrow-band power to wide-band power (see Ref. [30] p. 391), which is very similar to the MGPSR method. The Navigator receiver applies a low-pass filter to the output, which results in a less noisy measurement. A comparison of the $C / N_{0}$ outputs of the two receivers is shown in Fig. 23. A third-order polynomial was fit to the two data sets and the fit residuals were plotted to show the relative uncertainties in the two $C / N_{0}$ outputs. The standard deviation of the Navigator fit residuals was $0.224 \mathrm{~dB}$, and the standard deviation of the MGPSR fit residuals was $0.665 \mathrm{~dB}$.
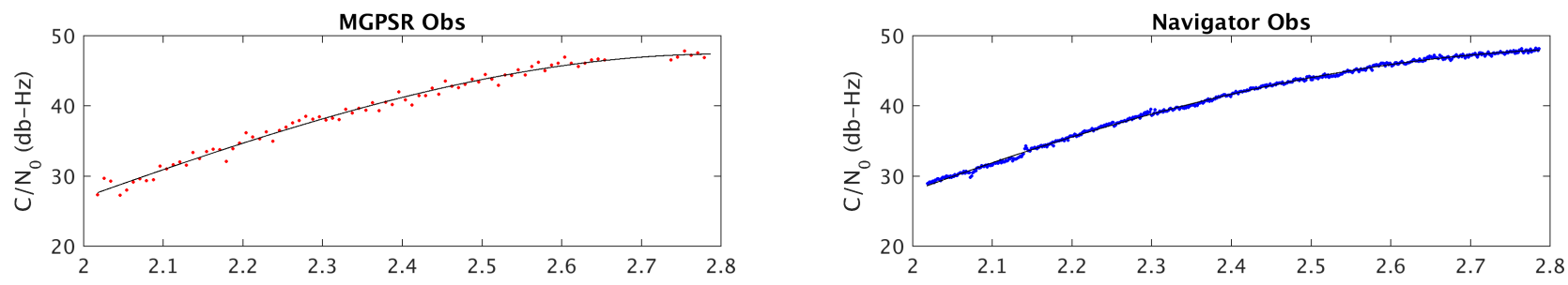

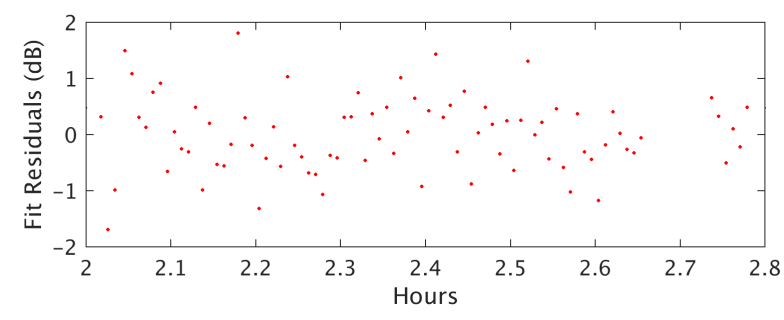

(a)

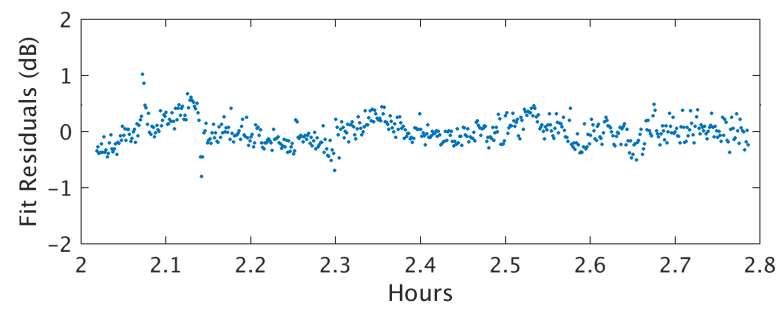

(b)

Figure 23: A comparison of MGPSR (a) and Navigator (b) received $C / N_{0}$ during one pass in the mainlobe with a third-order polynomial fit and fit residuals.

In addition to the variation in the $C / N_{0}$ estimate, there may also exist a mean error in the estimator output. While there is no way to determine if such an error exists by analysis of the GPS ACE data, there is a detailed discussion of the estimator performance in [32] which describes both the mean and standard deviation of the Navigator $C / N_{0}$ estimator as a function of $C / N_{0}$. As shown in [30] the error in the mean grows from approximately $0.25 \mathrm{~dB}$ at a $C / N_{0}$ of $30 \mathrm{~dB}-\mathrm{Hz}$ to $1.2 \mathrm{~dB}$ at $10 \mathrm{~dB}-\mathrm{Hz}$. For the GPS ACE data set, this effectively means that there is a greater uncertainty in the shape and depth of the null regions of the reconstructed antenna patterns than there is in the stronger main and side lobes. 


\subsubsection{Uncertainty in the Noise Temperature of the Receiver}

Another source of uncertainty in the GPS transmit antenna pattern reconstruction link budget is the calculation of the receiver noise density. The receive antenna noise temperature is a function of the integrated temperature over the entire aperture. This temperature is a function of the variation in temperature across the Earth disk and the changing attitude of the spacecraft. The contributions of the sun and moon in the antenna field of view were also ignored because it was found that, in the average, they did not change the noise temperature of the system significantly, and it was determined that the pre-binning data editing described in Section 4.4 removed the local outliers. Additionally, the total system noise temperature is a function of multiple elements, only the two largest of which were modeled: the LNA and the downconverter. This error is assumed to be fixed but is unknown. For example, an error in the system noise temperature of $25 \mathrm{~K}$ results in an error of approximately $0.6 \mathrm{~dB}$ in the calculation of noise density.

\subsubsection{Uncertainty in the Receiver Antenna Gain Pattern}

An additional source of uncertainty in the link budget is the uncertainty in the knowledge of the receive antenna pattern. The data available to the GPS ACE team were measurements of the antenna pattern at 9 elevation cuts around the azimuth (every $45 \mathrm{deg}$ ) from 0 to $45 \mathrm{deg}$ off-boresight angle at $3 \mathrm{deg}$ steps. The azimuthal variation in the pattern as a function of off-boresight angle increased to almost $10 \mathrm{~dB}$ at $45 \mathrm{deg}$ off boresight. This variation required interpolation of the antenna pattern data in two dimensions so that the actual receiver gain values could be computed for any received signal geometry. The original approach was a simple 2-dimensional interpolation in angle space. However, upon closer inspection, that method proved to have several flaws. First, because the antenna data is sparse in azimuth at the lower elevation angles, linear and spline interpolation were not accurate in those regions. Additionally, interpolation in angle space instead of Cartesian means the that information connecting data at 0 deg azimuth and $359 \mathrm{deg}$ azimuth was lost, creating larger errors in that region of the pattern. Instead, the antenna pattern data was converted from polar azimuth and elevation angles to Cartesian x,y pairs and then a surface was fitted to the data using a biharmonic gridded data method. This created a smooth surface to sample antenna gain at any received signal geometry as shown in Fig. 24.

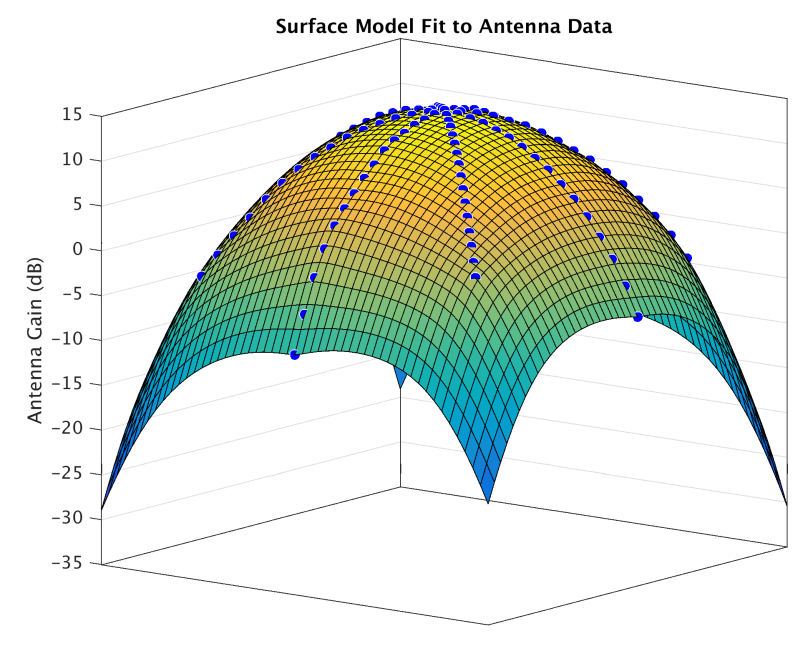

Figure 24: Smooth surface fitted to sparse receive antenna gain data.

\subsection{Other Error Sources}

Other error sources were considered in the verification process, including:

- Orbit/attitude ephemeris error 
- GPS ephemeris (almanac) error

- Orbit/attitude interpolation error

- GPS yaw error

- Coordination system transformation error (e.g. error in FK5 reduction)

- Floating-point round-off error

To evaluate the significance of these errors, the limits of acceptable error in a given measurement were determined based on an error threshold in the final link budget of $0.1 \mathrm{~dB}$ in gain and $0.5 \mathrm{deg}$ in transmit antenna azimuth/elevation position. These thresholds were chosen as error bounds to minimize impact to the end users. Using the link budget path loss and angular offset calculated at the smallest possible range between transmitter and receiver, the radial, in-track, and cross-track position errors were calculated that would cause a link budget error in excess of these thresholds. The calculated values were estimated to be well in excess of the expected combined error in all of the above sources.

The attitude error around boresight for both the receiver and transmitter is more directly mapped to an angular error on the final transmit pattern. These models were verified individually for correctness, and known intervals of greater uncertainty, such as GPS satellite shadow periods, were excluded. The presence of systematic angular biases was investigated during comparison with ground-test data at a pattern level, and none was found.

\section{Pseudorange Deviations}

The preceeding sections have discussed GPS signal availability for GEO and HEO applications. Next we address the signal quality at these high altitudes. Much work has been done for low-Earth orbit applications and for geodesy applications $[33,34]$. We also mention the SVN-49 signal anomaly because it provided an exceptional case of the variation of the signal delay with elevation [35]. In this paper, we use the term pseudorange deviation instead of group delay to address variations in the measured pseudorange with GPS boresight angle. The term pseudorange deviation has been introduced by Dybdal to address systematic differences between the pseudoranges determined from an actual GPS system and an ideal system [36]. The objective here is to assess systematic deviations in the pseudorange with the GPS boresight angle and azimuth at the large boresight angles in the SSV.

The expectation is that an assessment would be possible in the primary sidelobes of the shaped beam antenna pattern [37], and that the pseudorange deviations would be benign. However, outside of the primary sidelobes and in the nulls between the sidelobes, any measureable response would exhibit large deviations and have high levels of noise. In those regions, multiple scattering interactions would predominate, diminishing the utility of any detected signal.

\subsection{Approach}

The Aerospace high fidelity orbit determination tool TRACE is used with the post-fit ephemeris of the relay vehicle and National Geospatial-Intelligence Agency (NGA) precise phase-center ephemeris and clock for the GPS constellation to create pass-through pseudorange residuals for all observations. Residuals are edited and binned in the same manner as in the gain pattern processing, resulting in a mean residual in each 1 deg bin.

This mean residual is taken as an estimate of the pseudorange deviation $\delta_{P D}$ at each particular GPS off-boresight and azimuth angle.

If the average residual $\bar{b}$ in a bin can be expressed as

$$
\bar{b}=\frac{1}{n} \sum_{i=1}^{n}\left(\eta_{0}+\eta_{m}\right)+\delta_{P D}
$$

where $\eta_{0}$ and $\eta_{m}$ represent zero mean model and measurement errors, respectively, then for a sufficient number of samples the mean residual in a bin will be an estimate of the pseudorange deviation $\delta_{P D}$ at that boresight angle and azimuth. 

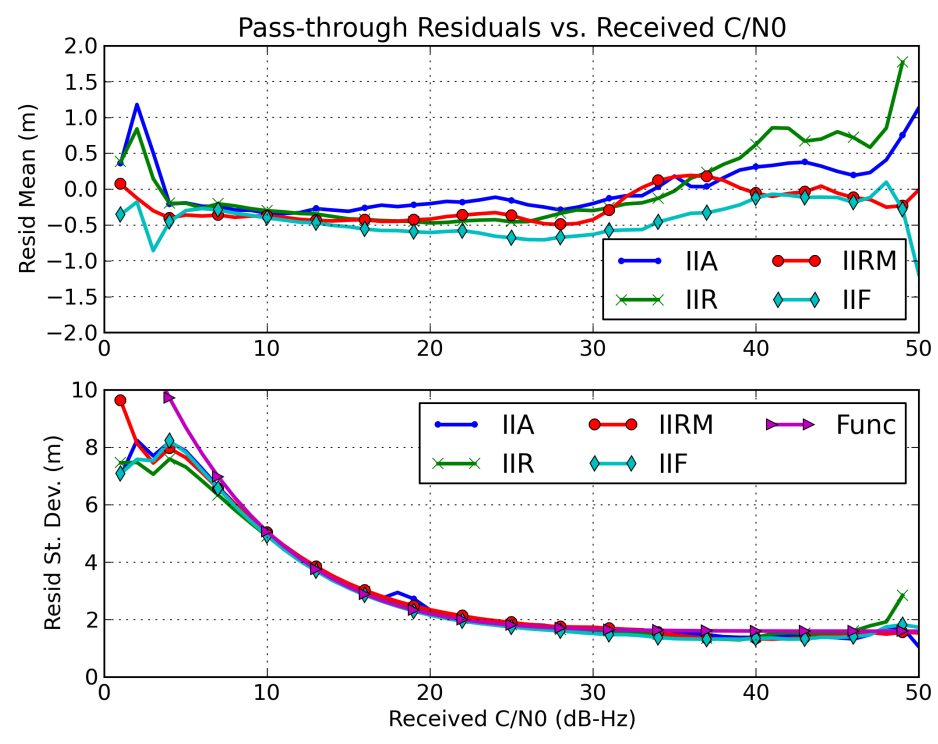

Figure 25: Pass-through pseudorange residual mean (top) and standard deviation (bottom) vs. received $C / N_{0}$.

Specific modeling compensation accounted for in TRACE includes GPS receive antenna and relay vehicle downlink antenna location offsets from the relay vehicle center of mass, relay vehicle attitude, precise ground station location, and satellite and ground path delays. The remaining effects such phase center variations of the L-band antenna panel shaped beam elements or element interaction are assumed to contribute to variations in the L-band signal causing systematic devations in the measured pseudorange. These deviations generally result from the GPS receiver correlator/discriminator response to distortions in the correlation function, and the response is dependent upon the correlator/discriminator design as discussed in Ref. [36]. In this regard, the MGPSR is a multitap receiver using many samples of the CA-code correlation function in each measurement and may not represent the performance of a early-late-prompt correlator receiver.

As stated above, the pseudorange residuals are computed using the satellite post-fit ephemeris, which does not use weak signal GPS observations in the orbit determination. This largely isolates the results from correlations due to fitting all the subject observations. In fact, post-fit residuals improve if TRACE is used to also solve for the ephemeris. However, since no weak signal GPS observations are used in the satellite ephemeris determination, residuals at large GPS off-boresight angles are not included in the minimization process and, thus, represent a fair assessment of the pseudorange deviation.

\subsection{Results}

\subsubsection{Residual Noise Correlation with Signal Strength}

Since the MGPSR can process the weakest signals, it is important to understand the correlation between pseudorange residual noise and received $C / N_{0}$. Figure 25 shows the mean (top) and standard deviation (bottom) of the raw pseudorange residuals versus received $C / N_{0}$ for each block based on the pass-through analysis. The values are created by sorting residuals into $1 \mathrm{~dB}-\mathrm{Hz}$ bins and taking sample statistics on each bin. The mean values have not been normalized, but they are generally within $1 \mathrm{~m}$ at all but the extreme signal levels. The spread in the mean at high signal levels is likely due to biases caused by atmospheric effects on main beam tranmissions.

The standard deviation plot shows that all blocks exhibit remarkably similar pseudorange noise. As expected, measurement noise increases as signal level decreases. A function can be determined to model the measurement 

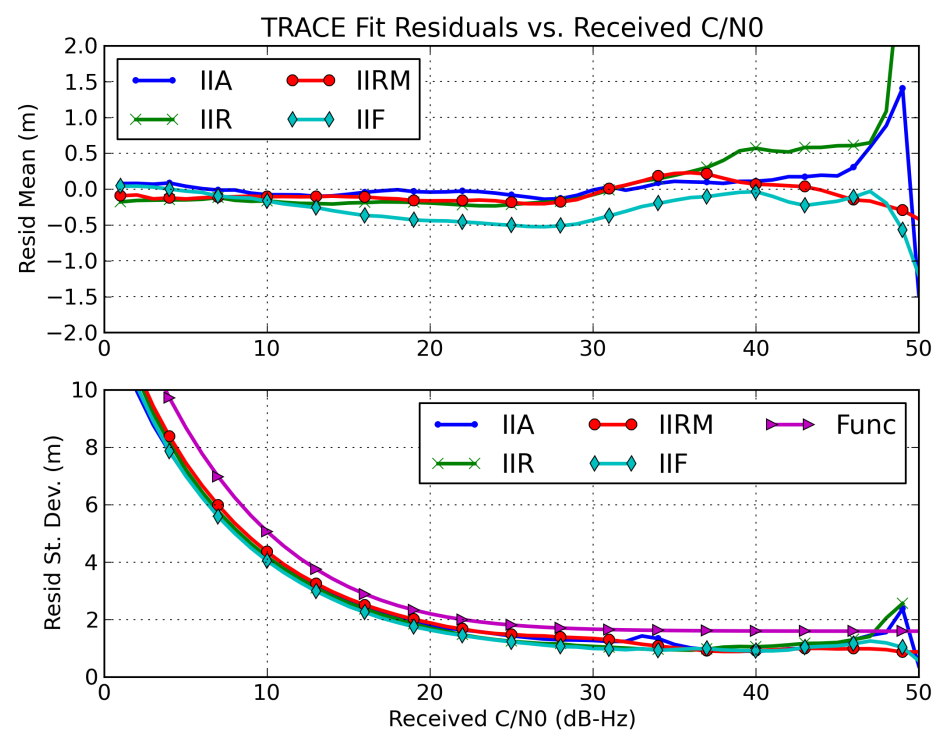

Figure 26: TRACE pseudorange residual mean (top) and standard deviation (bottom) vs. received $C / N_{0}$.

noise as a function of signal level - a useful tool for relative weighting of pseudorange in the orbit determination process. From Ref. [23], the relationship is

$$
\sigma_{P R}=1 \times 10^{-6} c\left[\frac{1}{1.8 B} \frac{1}{\sqrt{S N R}}\right]
$$

where $\sigma_{P R}$ is the pseudorange noise in $\mathrm{m}, c$ is the speed of light in $\mathrm{m} / \mathrm{s}, S N R$ is the signal-to-noise ratio of the received signal, and $B$ is the effective signal bandwidth in MHz. The $S N R$ is related to the $C / N_{0}$ through Eq. 2 where $S N R=P-1, T$ is the integration time in sec, and $C / N_{0}$ is expressed in $\mathrm{dB}-\mathrm{Hz}$.

The Func line in the bottom plot of Figure 25 is the effective pseudorange noise which is the RSS of the SNR component and a model component. The effective pseudorange noise $\sigma_{P R_{e f f}}$ can be modeled using a slight modification of Eq. 13, adding a bias to represent the noise floor,

$$
\sigma_{P R_{\text {eff }}}=\sqrt{\sigma_{P R}^{2}+\sigma_{\text {floor }}^{2}}
$$

where $\sigma_{\text {floor }}$ is an empirical noise floor determined from the data. In Figure 25, the $\sigma_{P R_{\text {eff }}}$ function is obtained using $\sigma_{\text {floor }}=1.6 \mathrm{~m}$ and $B=2 \mathrm{MHz}$ to produce an envelope of the pseudorange noise at the MGPSR integration time of $T=30 \mathrm{sec}$.

The residuals are reduced further by processing a subset of observations through Aerospace TRACE using only those observations with signal levels above $22 \mathrm{~dB}-\mathrm{Hz}$. Figure 26 shows the same quantities for the TRACE residuals as shown in Figure 25 for the pass-through analysis. The mean residuals show similar trends to those in Figure 25, but with slightly smaller offsets. The standard deviation plot shows the same $\sigma_{P R_{\text {eff }}}$ function as determined for the passthrough results, clearly demonstrating that the TRACE fit is able to reduce the noise floor. In this case, changing $\sigma_{\text {floor }}$ to $1.0 \mathrm{~m}$ in Eq. 14 would bring the effective noise function down to the level of the TRACE fit. Computing an independent trajectory estimation with strong side lobe signals and producing a similar, but lower noise fit provides confidence that the pass-through analysis represents a fair assessment of the pseudorange accuracy in the side lobes. To be more conservative, the remainder of the results in this section are generated from the pass-through analysis. 


\subsubsection{Block Average Performance}

The small biases in the raw residuals shown in Figure 25 are strong indicators of the expected pseudorange deviation performance. Binning the raw residuals into a $1 \mathrm{deg}$ by $1 \mathrm{deg}$ grid produces an estimate of the pseudorange deviation from that location in the GPS transmit pattern. Among many ways to view the results, samples are given here of pseudorange deviation behavior across constant elevation, constant azimuth, and averaged at each off-boresight angle.

Figure 27 shows cuts at constant off-boresight angles for Blocks IIR-M and IIF. The cuts occur at 18 (top), 30 (middle), and $45 \mathrm{deg}$ (bottom) off boresight. The $18 \mathrm{deg}$ cut is in the GPS main beam, while the others are in the side lobes. While there is some variation in the mean residual correlated to the location of nulls, the overall bias and variability are relatively small. Compare the azimuth of features in these plots to their respective gain plots in Figures 11 and 13.
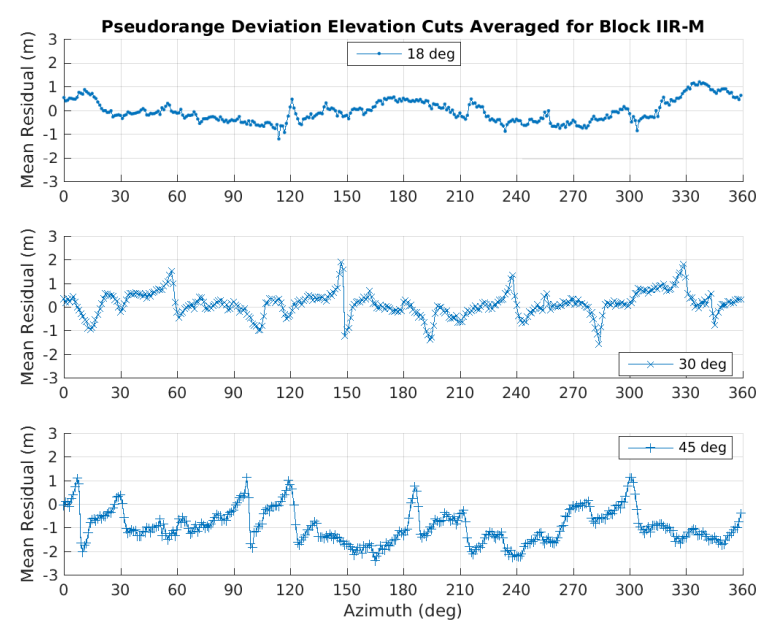

(a)
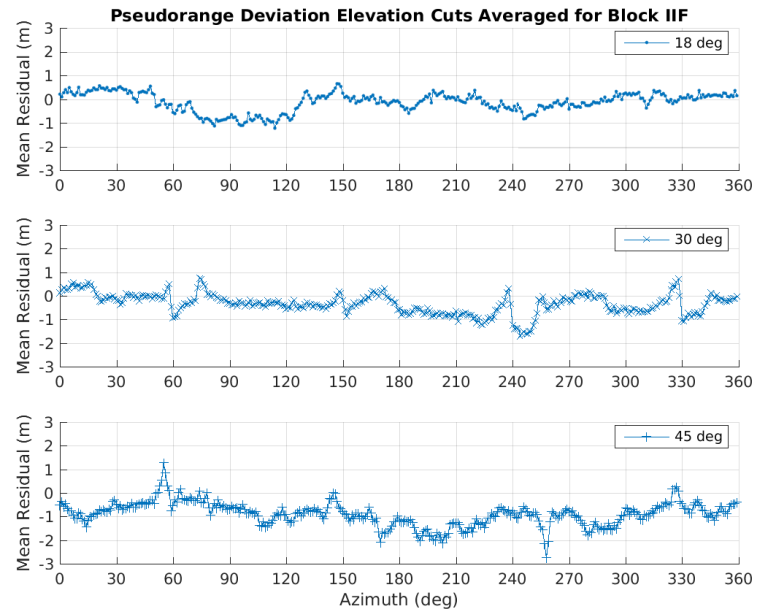

(b)

Figure 27: Elevation cuts at 18, 30, and 45 deg off boresight for Block IIR-M (a) and Block IIF (b) average pseudorange deviations.

Given that the elevation cuts show relatively small variations, normalization of the residuals by the mean across all azimuths at a location in the main beam provides a relative measure of the deviation in the side lobes, assuming small deviation in the outer portion of the main beam. The following results are normalized by removing the block-average residual at $18 \mathrm{deg}$ off boresight for each of the respective blocks. For example, the value removed for Block IIF is the mean of those values plotted in the top panel of Figure 27(b). The normalization constants for each block are shown in Table 5. Note that these are consistent with the biases at high $C / N_{0}$ values in the top plots of Figures 25 and 26.

Table 5: Mean pseudorange residual at $18 \mathrm{deg}$ off boresight for each block used for normalization. *The IIR-M category includes IIR SVNs with the modernized antenna panel.

\begin{tabular}{|l|c|}
\hline Block & $\begin{array}{c}\text { Mean Residual at } \\
\text { 18 deg off boresight (m) }\end{array}$ \\
\hline IIA & +0.36 \\
IIR & +0.58 \\
IIR-M* $^{*}$ & -0.05 \\
IIF & -0.13 \\
\hline
\end{tabular}

Using the normalized results, Figure 28 shows azimuth cuts every 15 deg out to 90 deg off boresight for Block IIR-M and IIF. In these plots, the mean at $18 \mathrm{deg}$ off boresight is zero. Clearly, some azimuths are noisier than others, again correlated to the signal levels. However, one feature that stands out is the general trend of all cuts towards a 
negative bias in the side lobes. Blocks IIA and IIR show similar trends.

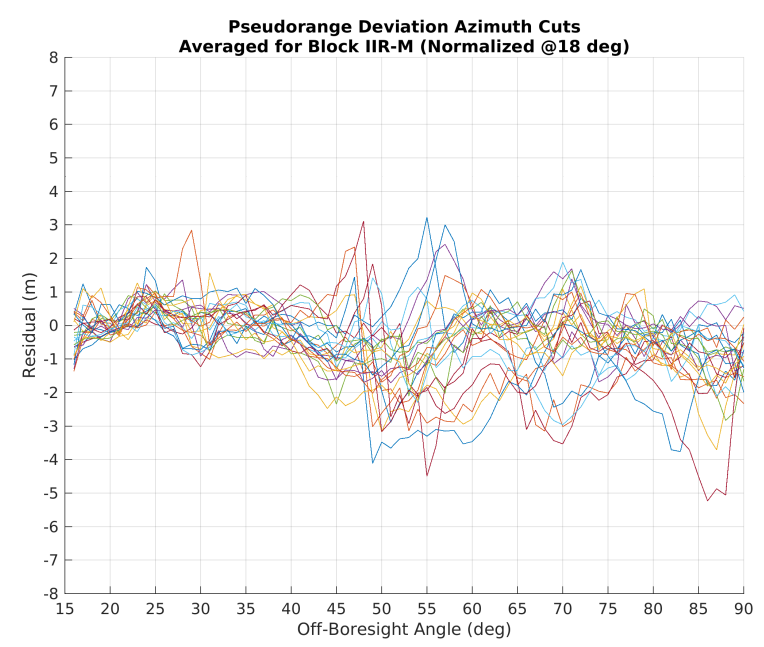

(a)

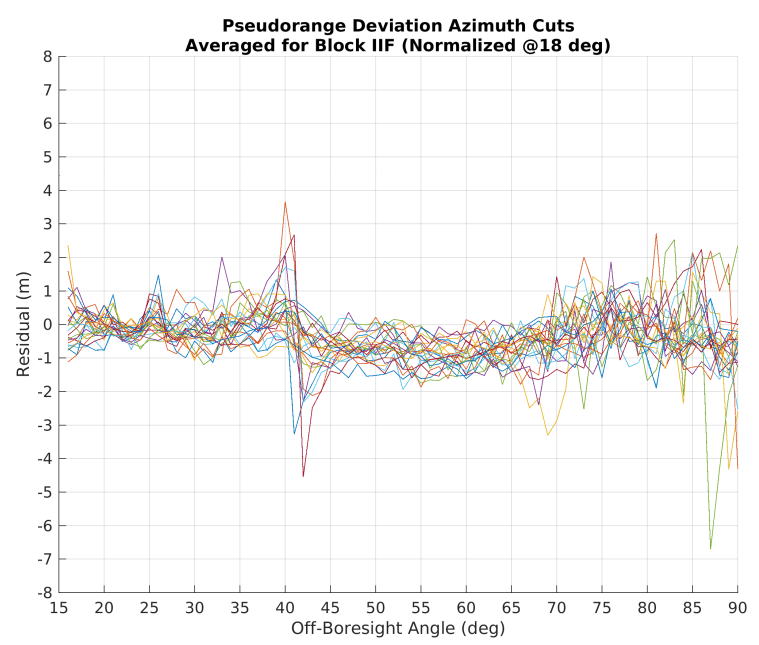

(b)

Figure 28: Azimuth cuts every 15 deg for Block IIR-M (a) and Block IIF (b) average pseudorange deviations.

Taking the mean at each elevation across all azimuths of the binned residuals for each of the four blocks results in Figures 29 and 30. The error bars represent the standard deviation of the means of the binned values at each off-boresight angle. Each figure has three subplots demonstrating the characteristics of the pseudorange deviation at different minimum received $C / N_{0}$ values: 0,15 , and $22 \mathrm{~dB}-\mathrm{Hz}$. For example, the middle plots in Figure 29 show the pseudorange deviation averaged over only those bins that have an average $C / N_{0}$ at or above $15 \mathrm{~dB}-\mathrm{Hz}$. If there are no bins at a given off-boresight angle that have a high enough signal level, nothing is plotted at that angle.

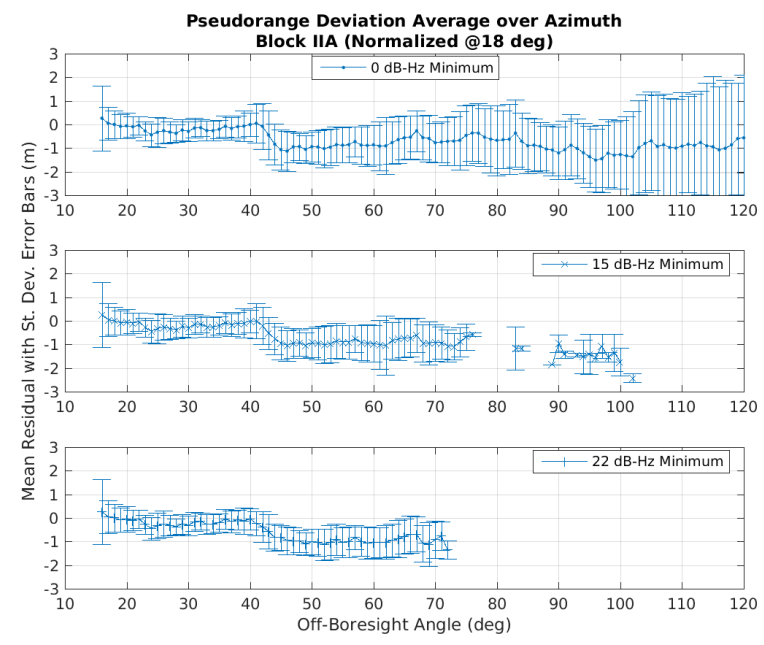

(a)

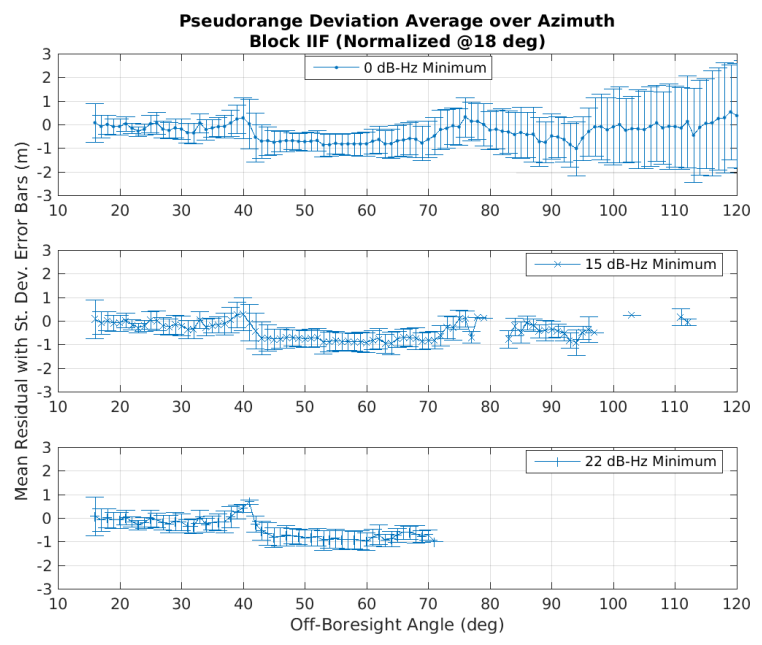

(b)

Figure 29: Block IIA (a) and Block IIF (b) pseudorange deviation mean over azimuth with standard deviation error bars.

Each block shows a similar trend of a negative bias in the side lobes that remains consistent at the different signal levels. The cause of the negative bias is unknown. It could be relay satellite ephemeris error, phase center variation of the GPS transmit antennas, or relay satellite receive antenna, a systematic effect in the TRACE pass through analysis, etc. However, the key feature of these plots is the small pseudorange deviation into the back lobes. For most high altitude GPS users, a deviation of 1-2 $\mathrm{m}$ is in the noise of the orbit determination solution or below the navigation accuracy requirement. This is clear evidence that pseudoranges in the GPS side lobes are of sufficient 


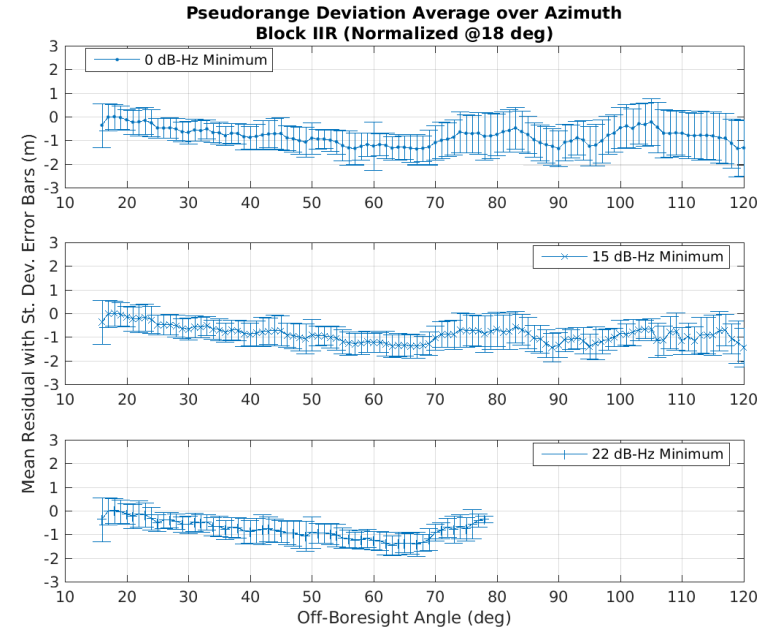

(a)

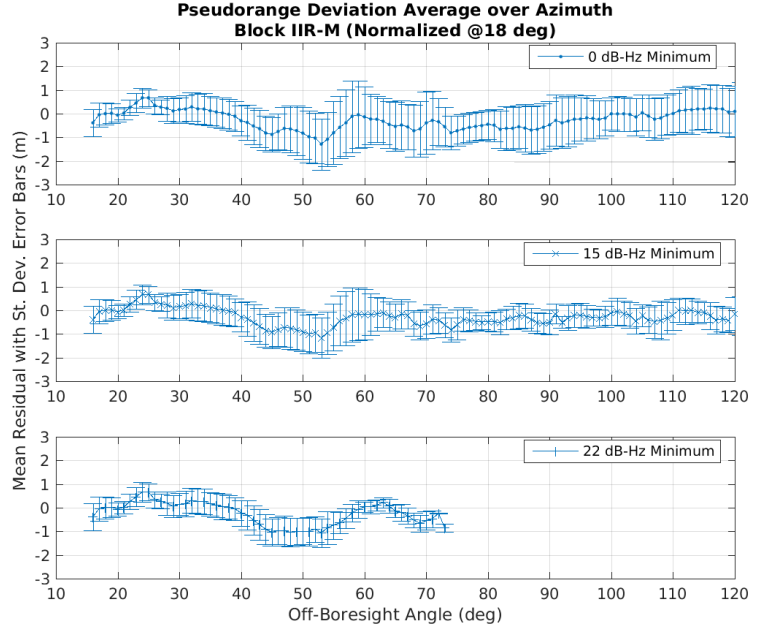

(b)

Figure 30: Block IIR (a) and Block IIR-M (b) pseudorange deviation mean over azimuth with standard deviation error bars.

quality to be useful for many HEO navigation applications. 


\section{Conclusions \& Future Work}

Extensive measurements of received GPS signal levels from a geosynchronous satellite recorded by the GPS Antenna Characterization Experiment have allowed comprehensive maps of GPS satellite gain and pseudorange deviations to be constructed for the entire GPS constellation. Fundamental to the collection of this dataset was the Aerospace Mariposa GPS Receiver and the long duration of the experiment. With sensitivity to below $0 \mathrm{~dB}-\mathrm{Hz}$, the MGPSR provides continuous all-in-view tracking from the GEO spacecraft, and the massive GPS ACE dataset provides many redundant measurements to enable statistical analysis and aid in identification of outliers.

Comparisons between gain measurements reconstructed from GPS ACE data and those measured in ground testing for the Block IIR and IIR-M satellites show excellent agreement. This demonstrates the value of conducting careful characterization of the GPS satellite antennas prior to launch because, even in the side lobes, these ground measurements have been demonstrated to be very representative of on-orbit performance. Furthermore, variations between individual GPS satellite gain patterns and the block-average patterns were found to be small outside of the deep null regions. This implies that analysts can safely use block-average gain patterns in their analysis and obtain realistic results.

This data set has provided insight into the pseudorange errors inherent in the side lobe signals, and has validated the suitability of side lobe transmissions for navigation. Range deviations constructed by generating pass-throughs of the measured pseudoranges with respect to the reconstructed orbit for the geosynchronous receiving spacecraft were found to be generally well below two meters. These results were obtained using a multi-tap correlator, and may vary with other receiver designs. Moreover, the range deviations exhibit a systematic pattern that would be conducive to applying a range deviation correction as a simple function of elevation to reduce the net error further.

The paper describes the extensive efforts taken through the post-processing of the data set to identify and remove erroneous measurements and other outliers, and to perform sensitivity analysis to characterize and quantify the various error sources inherent in the analysis of the data.

The GPS ACE reconstructed antenna patterns should allow precise modeling of GPS signal availability for high Earth orbit (HEO) users, whereas in the past mission planners had to make conservative assumptions regarding the availability of side lobe signals and their inherent error characteristics. This improved modeling fidelity is especially important for more stressing future HEO GPS applications such as use of GPS on cubesats, in which a directional receive antenna is likely precluded, or in applications to human lunar exploration of the moon [38]. Moreover this dataset will be invaluable to inform any changes that might be considered to GPS Space Service Volume (SSV) performance requirements in the future.

Looking to the future, the authors would like to further explore the extent of any temporal variations in transmitted power levels present in the data. There is also interest in utilizing the reconstructed antenna patterns and the ODTBX modeling software to compare observed vs. predicted GPS receiver power levels for operational missions such as GOES and MMS. As GPS III satellites are added to the GPS constellation in the near future, it is expected that GPS ACE data will be used in an on-orbit assessment of the performance of those satellites and to produce gain and pseudorange deviation data plots.

\section{Acknowledgment}

This work was performed under a collaborative Independent Research and Development effort between The Aerospace Corporation and NASA Goddard Space Flight Center authorized under Space Act Agreement SAA5-14-2-N16999.

\section{References}

[1] Winkler, S., Ramsey, G., Frey, C., Chapel, J., Chu, D., Freesland, D., Krimchansky, A., and Concha, M., "GPS Receiver On-Orbit Performance for the GOES-R Spacecraft," in 10th International ESA Conference on Guidance, Navigation $\&$ Control Systems, Salzburg; Austria, 2017. 
[2] Winternitz, L. B., Bamford, W. A., Price, S. R., Carpenter, J. R., Long, A. C., and Farahmand, M., "Global Positioning System Navigation Above 76,000 km for NASA's Magnetospheric Multiscale Mission," NAVIGATION, vol. 64, pp. 289-300, Summer 2017.

[3] Winternitz, L. B., Bamford, W. A., and Price, S. R., "Navigation Results from the Magnetospheric Multiscale Spacecraft and Simulations at Lunar Distances," in Proceedings of the 30th ION GNSS+, (Portland, OR), September 2017.

[4] Bauer, F., Moreau, M. C., Dahle-Melsaether, M., Petrofski, W., Stanton, B., Thomason, S., Harris, G., Sena, R., and Parker Temple III, L., "The GPS Space Service Volume," in Proceedings of the 19th International Technical Meeting of the Satellite Division of The Institute of Navigation (ION GNSS 2006), pp. 2503-2514, September 2006.

[5] Marquis, W. and Reigh, D., "The GPS Block IIR and IIR-M Broadcast L-Band Antenna Panel: Its Pattern and Performance," NAVIGATION, vol. 62, pp. 329-347, Winter 2015.

[6] Winternitz, L. M., Bamford, W. A., and Heckler, G. W., "A GPS Receiver for High-Altitude Satellite Navigation," IEEE Journal of Selected Topics in Signal Processing, vol. 3, August 2009.

[7] Martzen, P., Highsmith, D. E., Valdez, J. E., Parker, J. J., and Moreau, M. C., "GPS Antenna Characterization Experiment (ACE): Receiver Design and Initial Results," in Proceedings of the Institute of Navigation Joint Navigation Conference, June 2015.

[8] Jorgensen, P., "Autonomous Navigation of Geosynchronous Satellites Using the NAVSTAR Global Positioning System," in National Telesystems Conference, Galveston, TX, 1982.

[9] Wu, S. C., Yunck, T. P., Lichten, S. M., Haines, B. J., and Malla, R. P., "GPS Based Precise Tracking of Earth Satellites from Very Low to Geosynchronous Orbits," in National Telesystems Conference, 1992.

[10] Moreau, M. C., GPS Receiver Architecture for Autonomous Navigation in High Earth Orbits. PhD thesis, University of Colorado, 2001.

[11] Powell, T., Martzen, P. D., Sedlacek, S., Chao, C., Silva, R., Brown, A., and Belle, G., "GPS Signals in a Geosynchronous Transfer Orbit: Falcon Gold Data Processing," in Proceedings of the Institute of Navigation National Technical Meeting, pp. 575-585, January 1999.

[12] Balbach, O., Eissfeller, B., Hein, G. W., Zink, T., Enderle, W., Schmidhuber, M., and Lemke, N., "Tracking GPS Above GPS Satellite Altitude: First Results of the GPS Experiment on the HEO Mission Equator-S," in Proceedings of IEEE PLANS, pp. 243-249, 1998.

[13] Moreau, M., Davis, E., Carpenter, R., Davis, G., Jackson, L., and Axelrad, P., "Results from the GPS Flight Experiment on the High Earth Orbit AMSAT AO-40 Spacecraft," in Proceedings of the ION GPS 2002 Conference, Portland, OR, 2002.

[14] Kronman, J., "Experience Using GPS For Orbit Determination of a Geosynchronous Satellite," in Proceedings of the Institute of Navigation GPS, (Salt Lake City, UT), September 2000.

[15] Spilker, J. J., "GPS Signal Structure and Theoretical Performance," in Global Positioning System: Theory and Applications, vol. 1, ch. 3, Progress in Astronautics and Aeronautics, 1997.

[16] Czopek, F. M. and Shollenberger, L. S., "Description and Performance of the GPS Block I and II L-Band Antenna and Link Budget," in Proceedings of the International Technical Meeting of the ION Satellite Division, ION GPS, September 1993.

[17] Neumann, N., "In-Flight Results from the GPS Receiver on SmallGEO," in 68th International Astronautical Congress, (Adelaide, Australia), September 2017.

[18] Barker, L. and Frey, C., "GPS at GEO: A First Look at GPS from SBIRS GEO1," in Proceedings of the AAS Guidance Navigation and Control Conference, (Breckenridge, CO), February 2014. 
[19] Parker, J. J. K., Donaldson, J. E., Bauer, F. H., and Moreau, M. C., "Use and Protection of the GPS Side Lobe Signals for Enhanced Navigation Performance in High Earth Orbit," in American Astronautical Society Guidance, Navigation, and Control Conferece, Breckenridge, CO, AAS, February 2016.

[20] Unwin, M., Van Steenwijk, R. D., Blunt, P., Hashida, Y., Kowaltschek, S., and Nowak, L., "Navigating above the gps constellation preliminary results from the sgr-geo on giove-a," in Proceedings of the 26th International Technical Meeting of The Satellite Division of the Institute of Navigation (ION GNSS+ 2013), pp. 3305-3315, 2013.

[21] Verde, G., Unwin, M., Duncan, S., Hyslop, A., and Kowaltschek, S., "Revisiting the SGR-GEO on GIOVE-A for GPS Satellite Antenna Pattern Mapping," in GNC 2017: 10th International ESA Conference on Guidance, Navigation, and Control Systems, Salzburg, Austria, 2017.

[22] DiOrio, N. A. and Axelrad, P., "GPS Weak Signal Detection for Orbit Determination at Geosynchronous Altitudes." Discovery Learning Student Project Poster at CU-Boulder, April 2012.

[23] Stein, S., "Algorithms for Ambiguity Function Processing," IEEE Transactions on Acoustics, Speech, and Signal Processing, vol. ASSP-29, June 1981.

[24] Psiaki, M. L., "Block Acquisition of Weak GPS Signals in a Software Receiver," in Proceedings of the International Technical Meeting of the ION Satellite Division, ION GNSS, September 2001.

[25] Tsui, J. B. Y., Fundamentals of Global Positioning Systems Receivers, A Software Approach. J. Wiley and Sons, New York, 2000.

[26] CGSIC, "Limited Duration GPS C/A Power Testing." https://content.govdelivery.com/accounts/ USDHSCG/bulletins/182c419, 2017.

[27] NASA Goddard Space Flight Center, "Orbit Determination Toolbox (ODTBX)." http://sourceforge.net/ projects/odtbx/, 2015.

[28] Bar-Sever, Y. E., "A New Model for Yaw Attitude of Global Positioning System Satellites," The Telecommunications and Data Acquisition Progress Report 42-123, November 1995. Available at http://tda.jpl.nasa.gov/progress_report.

[29] "Radio Noise, Recommendation ITU-R P.372-13." http://www.itu.int/rec/R-REC-P.372-13-201609-I/en, 2016. International Telecommunications Union.

[30] Dierendonck, A. J. V., "GPS Receivers," in Global Positioning System: Theory and Applications (Parkinson, B. and Spilker, J., eds.), vol. 163 of Progress in Astronautics and Aeronautics, ch. 8, Washington, DC: AIAA, 1996.

[31] Jones, E., Oliphant, T., Peterson, P., et al., "SciPy: Open source scientific tools for Python, v. 0.7.2." http: //www.scipy.org/, 2001-2010.

[32] Winternitz, L., Bamford, W., and Heckler, G., "A GPS Receiver for High Altitude Satellite Navigation," IEEE Journal of Selected Topics in Signal Processing, vol. 3, no. 4, 2009.

[33] Wanninger, L., Sumaya, H., and Beer, S., "Group Delay variations of GPS transmitting and receiving antennas," J Geod, vol. 91, pp. 1099-1116, 2017.

[34] Schmid, R., Dach, R., Collilieux, X., Jaggi, A., Schmitz, M., and Dilssner, F., "Absolute IGS antenna phase center model igs08.atx: status and potential improvements," J Geod, vol. 90, pp. 343-364, 2016.

[35] Lake, J. and Stansell, T., "SVN-49 Signal Anomaly," in 49th meeting of the Civil GPS Service Interface Committee (CGSIC), (Savanna, GA), September 2009. https://www.gps.gov/cgsic//meetings/2009/stansell.pdf.

[36] Dybdal, R. B., "GPS Satellite Pseudorange Deviation Contributions," Aerospace Report No. TOR-2014-02719, The Aerospace Corporation, 2014. 
[37] Brumbaugh, C., Love, A., Randall, G., Waineo, D., and Wong, S., "Shaped Beam Antenna for the Global Positioning Satellites," in IEEE AP-S Symposium Digest, (Amherst, MA), October 1976.

[38] Ashman, B. W., Parker, J. J. K., Bauer, F. H., and Esswein, M., "Exploring the Limits of High Altitude GPS for Lunar Missions," in American Astronautical Society Guidance, Navigation, and Control Conferece, Breckenridge, CO, AAS, February 2018. 